\title{
Modelos Estocásticos e Propriedades Estatísticas em Mercados de Alta Frequência
}

\author{
Helder Alan Rojas Molina \\ DisSERTAÇAÕ APRESENTADA \\ $\mathrm{AO}$ \\ Instituto de Matemática e Estatística \\ DA \\ UNIVERSIDADE DE SÃo PAUlo \\ PARA \\ OBTENÇÃO DO TÍTULO \\ DE \\ Mestre em CiÊnCIAS \\ Programa: Estatística \\ Orientador: Prof. Dr. Anatoli Yambartsev
}

Durante o desenvolvimento deste trabalho o autor recebeu auxílio financeiro da CAPES e CNPq.

São Paulo, dezembro de 2015 


\section{Modelos Estocásticos e Propriedades Estatísticas em Mercados de Alta Frequência}

Esta é a versão preliminar da dissertação elaborada pelo candidato Helder Alan Rojas Molina. 


\section{Resumo}

\section{H. Rojas, Modelos Estocásticos e Propriedades Estatísticas em Mercados de Alta Frequên-}

cia. 2015.

Dissertaçaõ (Mestrado) - Instituto de Matemática e Estatística, Universidade de São Paulo, São Paulo, 2015.

Neste trabalho, apresentamos um conjunto de fatos empíricos e propiedades estatística de negociações em alta frequência, e discutimos algumas questões gerais comuns a dados de alta frequência tais: como discretização, espaçamento temporal irregular, durações correlacionadas, periodicidade diária, correlações temporaise e as propriedades estatísticas dos fluxos de ordens.

Logo apresentamos dois modelos da literatura,estilizados para a dinâmica do limit order book. No primeiro modelo o fluxo de ordens é descrito por processos de Poisson independentes, propomos para ele uma forma alternativa da prova de ergodicidade baseada em funções de Lyapunov. O segundo modelo é um modelo reduzido que toma em consideração dinâmicas tipo difusão para os tamanhos do bid e ask, e centra o foco só nas ordens como melhor preço, e modela explicitamente as cotações do bid e ask na presença de liquidez oculta.

E por ultimo, propomos um modelo alternativo para a dinâmica do preço eo spread no limit order book, estudamos o comportamento assintótico do modelo e estabelecemos condições de ergodicidade e transitoridade. Além disso, consideramos a uma família de cadeias de Markov definidos nas sequências de caracteres (strings, ou palavras) com infinito alfabeto e para alguns exemplos inspirados nos modelos de negociações em alta frequência, obtemos condições para ergodicidade, transitoriedade e recorrência nula, para a qual usamos as técnicas de construção de funções Lyapunov.

Palavras-chave: limit order book, dados de alta frequência, processos de Poisson, fluxos de ordens, bid e ask, funções Lyapunov, liquidez oculta. 


\section{Abstract}

H. Rojas, Stochastic Models and Statistical Properties in High Frequency Markets. 2015. Dissertation (MSc.) - Instituto de Matemática e Estatística, Universidade de São Paulo, São Paulo, 2015 .

In this work, we present a set of empirical facts and statistical properties of negotiations at high frequency and discuss some general issues common to high-frequency data such: as discretization, irregular spacing, correlated durations, daily periodicity, temporaise correlations and the statistical properties of flows orders.

Soon we present two models stylized in the literature for the dynamic limit order book. In the first model the order flow described by separate Poisson processes and we propose it to an alternative form of test ergodicity based on Lyapunov function. The second model is a reduced model that takes into consideration diffusion-type dynamics for the sizes of the bid and ask, and centers focus only on orders as best price and model explicitly quotes the bid and ask in the presence of hidden liquidity.

And finally, we propose an alternative model for the price dynamics and spread in the limit order book, we study the asymptotic behavior of the model and established conditions of ergodicity. Furthermore, we consider the a family of Markov chains defined on the sequences of characters (strings, or words) with infinite alphabet. For some examples inspired by the models of high frequency trading we obtain a conditions for ergodicity, transience and null-recurrence. In order to prove this we use the construction of Lyapunov functions techniques.

Keywords: limit order book, high frequency data, Poisson processes, order flows bid and ask, Lyapunov functions, hidden liquidity. 


\section{Sumário}

$\begin{array}{ll}\text { Lista de Figuras } & \text { ix }\end{array}$

$\begin{array}{lll}\mathbf{1} & \text { Introdução } & \mathbf{1}\end{array}$

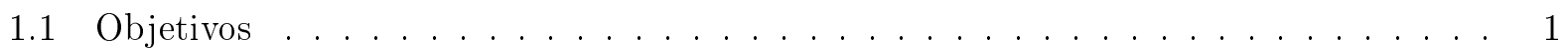

1.2 Organização do Trabalho . . . . . . . . . . . . . . . . . . . . 2

2 Fatos empíricos e propriedades estatísticas em mercados de alta frequência 3

2.1 Mercados de alta frequência $\ldots \ldots \ldots \ldots \ldots \ldots \ldots$

2.2 Dados de alta frequência $\ldots \ldots \ldots \ldots \ldots \ldots \ldots \ldots$

2.3 Fatos empíricos em alta frequência $\ldots \ldots \ldots \ldots \ldots \ldots \ldots$

2.4 Fluxo de ordens . . . . . . . . . . . . . . . . . . . . . . . . 8

$2.4 .1 \quad$ Propriedades estatísticas dos fluxos de ordens $\ldots \ldots \ldots \ldots$

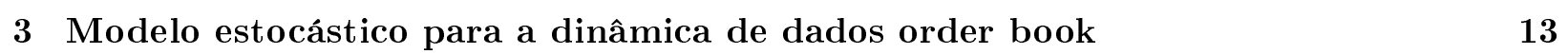

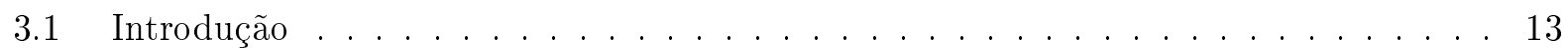

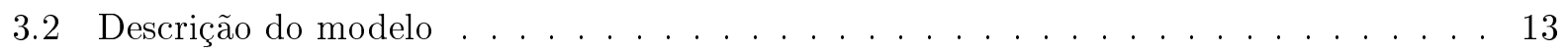

3.3 Suposições do modelo $\ldots \ldots \ldots \ldots \ldots \ldots \ldots$. . . . . . . . . . . . . . . . 14

3.4 Dinâmica do order book . . . . . . . . . . . . . . . . . . . . . . . . . 14

3.5 Transformadas de Laplace para calcular probabilidades condicionais. . . . . . . . . . 15

3.5 .1 Tempos de primeira passagem de processos nascimento-morte . . . . . . . 15

3.5 .2 Direção de movimentos de preços . . . . . . . . . . . . . . . 16

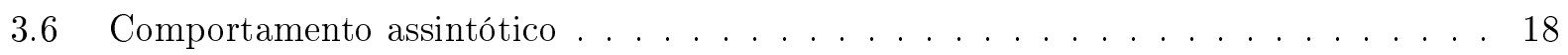

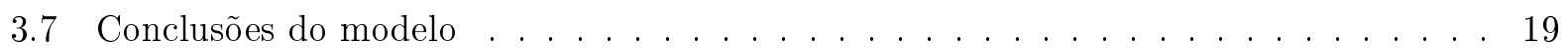

4 Previsão de Preços para dados de nível-I na presença de liquidez oculta 21

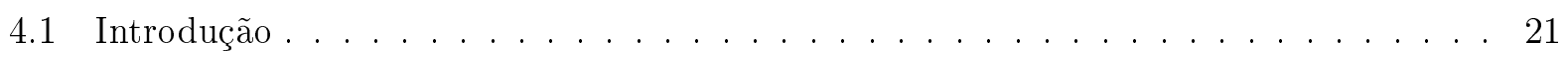

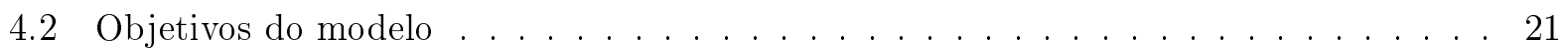

4.3 Modelando cotações de nível-1 . . . . . . . . . . . . . . . . . . . 22

4.4 A difusão limite . . . . . . . . . . . . . . . . . . . . . . . . . . . . . . . 23

4.5 A equação diferencial parcial $\ldots \ldots \ldots \ldots \ldots \ldots \ldots \ldots$

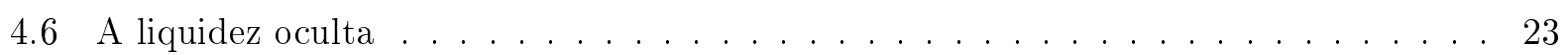

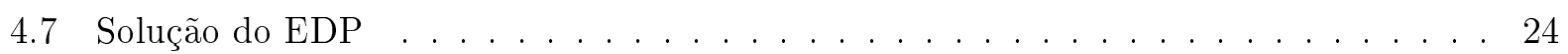

4.7 .1 Teorema . . . . . . . . . . . . . . . . . . . . . . . . . . . . . 24

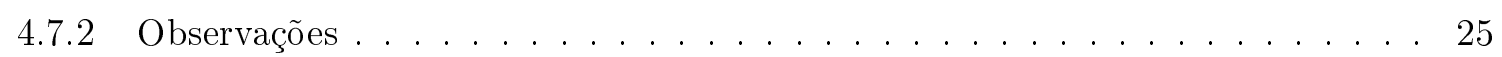

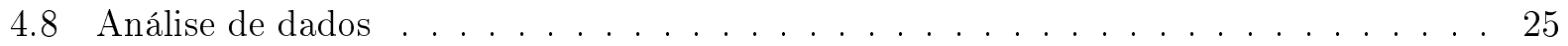


4.9 Concluções do modelo $\ldots \ldots \ldots \ldots \ldots \ldots \ldots$

5 Dinâmica do Spread bid-ask no limit order book

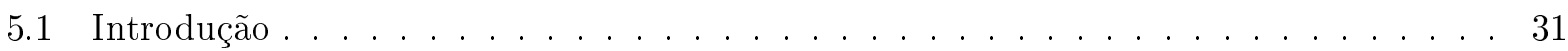

5.2 Definição do modelo . . . . . . . . . . . . . . . . . . . . . . 31

5.3 Dinâmicas dos preços bid e ask . . . . . . . . . . . . . . . . . . 32

5.3 .1 Taxas de transição uniforme . . . . . . . . . . . . . . . . . . 32

5.3 .2 Taxas de transição com decaimento constante . . . . . . . . . . . . . . . . . 34

5.3 .3 Taxas de transição com decaimento polinomial . . . . . . . . . . . . . . 35

6 Modelos simples de sequências de caracteres com infinito alfabeto $\quad 37$

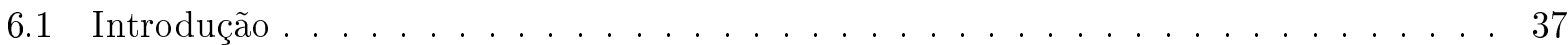

6.2 Descrição do modelo . . . . . . . . . . . . . . . . . . . 37

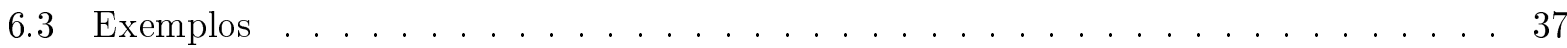

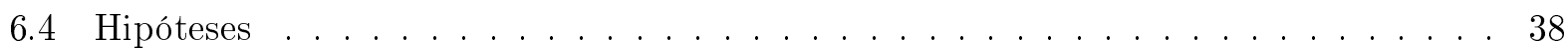

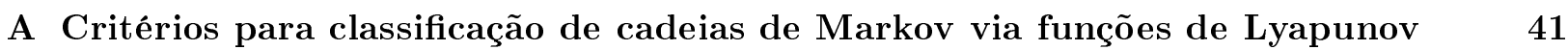

\begin{tabular}{ll}
\hline Referências Bibliográficas & 43
\end{tabular} 


\section{Lista de Figuras}

2.1 Representação gráfica da dinâmica do order book das ações de AstraZeneca negociadas na Bolsa de Londres, durante um intervalo de 10 min em 4 de setembro de 2002.

Os círculos preenchidos(vazios) são operações de compra (venda). As linhas grossas

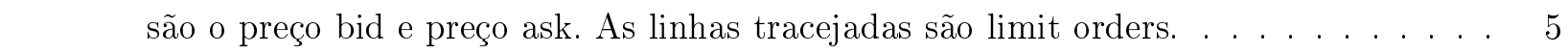

2.2 Quantis de durações comparados com quantis de uma distribuição exponencial com a mesma média (Citigroup, Junho de 2008). A linha tracejada representa os quantis de uma distribuição exponencial, que se encontram bastante diferentes dos quantis

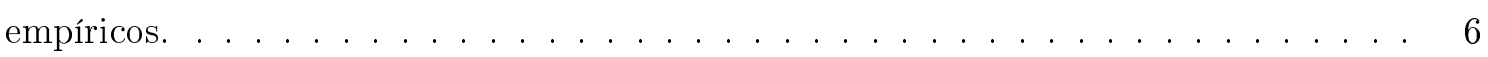

2.3 Exemplos de padrão intra-diário que registra 15 minutos de negociações eo eixo $x$ mostra a hora do dia. A figura mostra o volume negociado (a), variações de preço absoluto (b), eo número de negociações (c) da General Electric na NYSE durante o período de 2002-2003. (d) O número de negociações da AstraZeneca trocados no mercado eletrônico (SET1) do LSE durante o período de maio 2000 a dezembro de

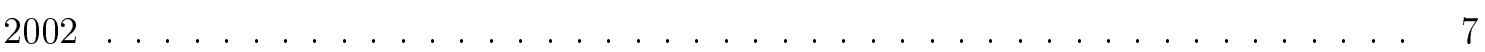

2.4 Função de autocorrelação dos retornos dos preços de transacção (círculos sólidos), valor absoluto dos retornos dos preços de transacção (círculos abertos), os retornos de preços médios amostradas antes de cada transação (triângulos a cheio), e retornos dos preços médios amostrados antes de cada evento de mercado (quadrados preenchidos) para AstraZeneca (AZN) ações negociadas na LSE em 2002. Os desfasamentos na linha horizontal são medidos em tempo do evento. . . . . . . . . . . . . . 7

2.5 Um limit order de compra: comprar dois em $69.200 . \ldots \ldots \ldots$. . . . . . . . 8

2.6 Um market order de venda de $10 . \ldots \ldots \ldots \ldots \ldots$. . . . . . . . . . . . . . 8

2.7 Função densidade de probabilidade do log-volume de ordens individuais, para os três componentes diferentes do fluxo de ordens. Para efeito de comparação, a figura também mostra a função densidade de probabilidade de logaritmo decimal do volume no melhor preço (ask), amostrados antes de cada transação. A inserção mostra em uma escala logarítmica dupla a distribuição de probabilidade acumulada do volume para os diferentes tipos de ordnes. A linha pontilhada é uma função polinomial com inclinação de 2,8, que é o estimador de máxima verossimilhança do expoente de cauda para os order market. Os dados referem-se ao AZN ações negociadas na LSE no período 1999-2002. . . . . . . . . . . . . . . . . . 10 
2.8 Autocorrelation dos fluxos de ordens plotados em uma escala logarítmica dupla. Especificamente, os painéis mostram a função de autocorrelação do market order (a), volume dos market orders (b), limit orders (c), e cancelamentos (d). Os dados referem-se ao AZN ações negociadas na LSE no período 1999-2002 $\ldots$. . . . . . . . . 10

2.9 Taxas de diferentes tipos de eventos no limit order book condicionados ao tamanho do spread. Os dados referem-se a ações de AZN negociados na LSE em 2002. . . . . . 11

4.1 As probabilidades empíricas para movimento ascendente dos preços (XLF em NAS-

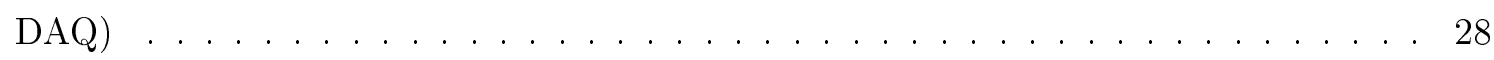

4.2 Probabilidades teóricas do modelo ajustado (XLF em NASDAQ) $\ldots \ldots \ldots$. . . . . . 28

4.3 Diferença entre as probabilidades empíricas e ajustadas . . . . . . . . . . . . . . . 29 


\section{Capítulo 1}

\section{Introdução}

A evolução dos preços nos mercados financeiros resulta da interação de comprar e vender ordens através de um processo dinâmico bastante complexo e em muitos casos isso acontece a grandes velocidades. Os estudos sobre os mecanismos envolvidos nas negociações de ativos financeiros têm tradicionalmente focados em plataformas eletrônicas de negociações na qual compra e vende ordens e fornece liquidez colocando cotações bid e ask. O sistema especialista NYSE é um exemplo deste mecanismo. Estas plataformas eletrônicas agregam todas os limit order pendentes no limit order book que está disponível para os participantes do mercado e os market order são executadas contra os melhores preços disponíveis no limit order book. O grande volume de dados disponíveis, a presença de regularidades estatísticas nos dados ea natureza mecânica de execução das ordens torna os mercados de alta frequência em candidatos interessantes para análise estatística e modelamento estocástico. Uma motivação importante para a modelagem da dinâmica de alta frequência do order book é entender o processo de evolução de preços como resultado da interação dos fluxos de ordens, e usar a informação sobre o estado atual do order book a fim de prever seu comportamento de curto prazo.

Modelos de equilíbrio de formação de preços em mercados de alta frequência [( $(\overline{\mathrm{PC}} 98),(\overline{\mathrm{RI}} 09)]$ mostraram que a evolução do preço nesses mercados é bastante complexo e depende do estado do order book. Por outro lado, estudos empíricos sobre limit order book [( $(\overline{\mathrm{BFL}} 08),\left(\overline{\mathrm{FGL}^{+} 04}\right)$, (GCJG99), ( HBMS), (SFGK03)] fornecem uma extensa lista de características empíricas e estatísticas da dinâmica do order book que são difíceis de incorporar em um único modelo.

A busca por modelos de mercados de alta frequência têm levado ao desenvolvimento de modelos estocásticos cujo objectivo é reter as principais características estatísticas dos limit order book, que por sua vez devem ser computacionalmente tratáveis e eficientes. Estes modelos estocásticos também servem para ilustrar o quão longe se pode ir em reproduzir as propriedades dinâmicas do limit order book, sem recorrer a supostos comportamentais detalhados sobre os participantes do mercado ou a introdução de parâmetros não observáveis que descrevam as preferências dos agentes, como nos modelos mais detalhados de microestrutura de mercado.

\section{$1.1 \quad$ Objetivos}

A partir da descrição clássica de chegadas de ordens e cancelamentos como processos pontuais, na literatura a dinâmica do limit order book é descrito na linguagem da teoria das filas. Entre os modelos mais recentes e relevantes nós consideramos dois; o modelo proposto por Cont, Stoikov e Talreja (CST10) eo modelo proposto por Avellaneda, Reed e Stoikov (ARS10). No primeiro modelo, a dinâmica do limit order book é modelado como um sistema de filas multi-classe, e usando métodos de transformada de Laplace, calculam várias probabilidades de transição do preço condicionais ao estado do order book. No segundo modelo, os autores consideram um modelo reduzido, se concentram apenas nas filas com melhores preços e propõem uma dinâmica de difusão para a evolução dos tamanhos dessas filas. 
O primeiro objectivo deste trabalho é fazer uma revisão deses modelos mencionados acima e provar sua ergodicidade usando técnicas de construção de funções Lyapunov [ver Apêndice A]. Um segundo objectivo é propor um modelo opcional para a dinâmica do order book baseado apenas na dinâmica Markoviana dos preços bid e ask. Um tecer objetivo é apresentar um conjunto de hipóteses sobre uma família de cadeias de Markov, definidas numa sequencia de caracteres com alfabeto infinito, que foram inspirados pelas negociações de alta frequência.

\subsection{Organização do Trabalho}

No Capítulo 2, apresentamos um conjunto de fatos empíricos estilizados que emerge da análise estatística de dados de alta frequência em vários tipos de mercados financeiros. Discutimos primeiro algumas questões gerais comuns a dados de alta frequência tais como: discretização, espaçamento temporal irregular, durações correlacionadas, periodicidade diária e correlações temporais. Logo são descritos as propriedades estatísticas dos fluxos de ordens para cada componente separadamente: maket order, limit order e cancelamentos. No Capítulo 3 apresentamos um modelo estilizado para a dinâmica do limit order book, na qual o fluxo de ordens é descrito por processos de Poisson independentes, ao mesmo tempo propomos uma forma alternativa da prova de ergodicidade do modelo baseada em funções de Lyapunov. Também mostramos como este modelo pode ser usado para calcular as probabilidades condicionais de vários tipos de eventos relevantes para a execução de negociações como por exemplo um movimento ascendente do preço, usando métodos de transformadas de Laplace. No Capítulo 4 será apresentado um modelo reduzido que toma em consideração dinâmicas tipo difusão para os tamanhos do bid e ask, e centra o foco só nas ordens como melhor preço (dados de nível I). Em contraste com o modelo anterior, este modela explicitamente as cotações do bid e ask na presença de liquidez oculta, ou seja os tamanhos que não são mostrados no order book mas podem influenciar nas probabilidades de um movimento ascendente do preço. Nosso primeiro aporte encontra-se no Capítulo 5 Neste capítulo, propomos um modelo alternativo para a dinâmica do preço eo spread no limit order book, estudamos o comportamento assintótico do modelo e estabelecemos condições de ergodicidade e transitoridade. Finalmente, no Capítulo 6 consideramos a uma família de cadeias de Markov definidos nas sequências de caracteres (strings, ou palavras) com infinito alfabeto. Para alguns exemplos inspirados nos modelos de negociações em alta frequência, obtemos condições para ergodicidade, transitoriedade e recorrência nula. Para provar isso, usamos as técnicas de construção de funções Lyapunov. No Apêndice A, apresentamos critérios para classificação de cadeias de Markov via funções de Lyapunov. 


\section{Capítulo 2}

\section{Fatos empíricos e propriedades estatísticas em mercados de alta frequência}

Neste capitulo presentamos um conjunto de fatos empíricos estilizados que emerge da análise estatística de dados de alta frequência em vários tipos de mercados e intrumentos financeiros. Discutimos algumas questões gerais comuns a todos os estudos estatísticos de dados de alta frequência e apresentamos propriedades estatísticas dos fluxos de ordens.

\subsection{Mercados de alta frequência}

Nos últimos anos, as plataformas eletrônicas de negociações têm substituído amplamente ao piso de negociões nos mercados acionários, tais como NYSE, Nasdaq, Tokyo Stock Exchange e London Stock Exchange (LSE). Em contraste com os mercados na qual um market maker ou especialista centraliza as ordens e fornece liquidez através de colocação das ordens de compra e venda, estas plataformas eletrônicas agregam todas os limit order pendentes no limit order book que está disponível para os participantes do mercado, e os market order são executadas contra os limit orders com melhores preços disponíveis no mercado, tudo isso é feito de uma maneira mecânica ea grandes velosidades, a frequência com que as ordens chegam no mercado aumentou eo tempo de execução de ordens caiu de mais de 25 milissegundos a 1 milissegundo nos últimos dez anos.

Como resultado, a evolução da oferta, demanda e comportamento dos preços nos mercados de ações está sendo cada vez mais registradas: esses dados estão disponíveis para os participantes do mercado no tempo real, em forma de bases de dados de alta frequência. A análise de dados de alta frequência constitui um desafio devido a seu grande volume e complexidade. Estes dados nos fornecem uma visão detalhada do processo dinâmico complexo através do qual o mercado "digere"os

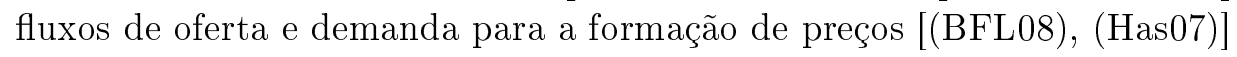

O grande volume de dados disponíveis, a presença de regularidades estatísticas nos dados ea natureza mecânica de execução das ordens torna os mercados de alta frequência em candidatos interessantes para análise estatística e modelamento estocástico. Em um nível fundamental, análise estatística e modelagem estocástico de dados de alta frequência pode fornecer conhecimento sobre a

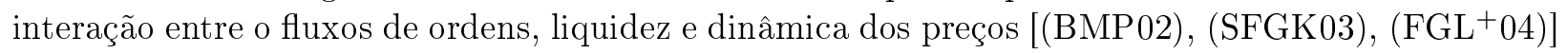
e pode ajudar a preencher a lacuna entre a teoria de microestrutura de mercado [( $(\mathrm{AB} 98),(\overline{P a r} 98)]$ e modelos estocásticos usado em gestão de riscos, estrategias de negociação e regulação do mercado, que representam o preço como um processo aleatório exógeno. 


\subsection{Dados de alta frequência}

Dados financeiros de alta frequência geralmente se referem a dados coletados em um horizonte muito curto de tempo. Nesse sentido, os dados de alta frequência essencialmente são concebido como dados intra-diários. No entanto, o significado de alta frequência mudou ao longo dos anos seguintes, cresceu a disponibilidade de informação mais e mais detalhada acerca do processo de negociação. Um ponto de vista mais rigorosa é considerar os registros financeiros onde nenhum tipo de agregação temporal é feito. O uso desses dados em finanças é recente e remonta ao final dos anos 1980. Entre as primeiras bases de dados de alta frequência são os dados de opção de Berkeley, contendo o preço bid eo preço ask (maior preço de compra e menor preço de venda disponiveis no order book) e preços de opção negociadas na Chicago Board Option Exchange (CBOE), o base de dados TORQ contendo negociações na New York Stock Exchange (NYSE 1990-1991), o base de dados HFDF93 divulgados pelo Olsen \& Associates (1992 a 1993). A seguir, descrevemos os tipos mais comuns de conjuntos de dados.

\section{- Dados tick-by-tick}

A forma mais simples de dados financeiros sem nenhuma agregação temporal é os chamados dados tick-by-tick. Neste tipo de dados, todas as operações são registradas. Normalmente, o conjunto de dados contém informações sobre o tempo, preço e volume de cada negociação. Claramente, a frequência de tempo em que os dados são gravados não é fixo, mas pode ser diferente em diferentes fases de um dia de negociação.

\section{- Dados de nível-I}

Também chamado dados Trade and quote, é um tipo mais refinado de conjunto de dados, contém informações sobre negociações e as melhores cotações. Neste tipo de dados geralmente contêm os preços de transação, o tempo quando a transação ocorreu, eo volume negociado. Dados de nível-I normalmente só contêm informações sobre o preço bid eo preço ask e do volume disponível nesses preços. Estes dados geralmente são atualizados cada vez que há uma mudança nos preços ou volume. Um dos dados de nível-I mais famosos é o banco de dados TAQ (trade and Quote), mantido pela NYSE desde 1993.

\section{- Dados order book}

O próximo nível de resolução de dados de alta frequência são os dados order book ou também chamados dados de nivel-II, os dados order book contém informação completa do order books num determinado mercado. Enquanto os dados de nível-I contém informação apenas sobre os preços bid e ask, os dados order book contém a informação de todos os limit order de compra e venda os qualés são gravados no order book quando eles são colocados e são tirados do order book quando eles expiram ou são cancelados. Este tipo de conjunto de dados permite reconstruir o limit order book em qualquer instante do tempo e de acompanhar a dinâmica do mercado mais de perto. Uma das bases de dados mais populares de dados order book é o Rebuild Order Book mantido pela London Stock Exchange (LSE).

\subsection{Fatos empíricos em alta frequência}

Os dados de alta frequência registram as negociações que ocorrem num mercado de ações, cada registro é associado com uma sinal (compra ou venda), tempo específico, preço de cotação e uma quantidade (volume da operação em número de ações). Os dados de alta frequência apresentam diversas particularidades que podem ser classificados da seguente forma [(Has07), (Hua04)].

\section{- Discretização}

Os preços dos ativos só podem assumir múltiplos inteiros de um 'tick'- $\delta$ - que é a menor variação de preço permitido. Da mesma forma, o volume de uma ordem deve ser múltiplo 


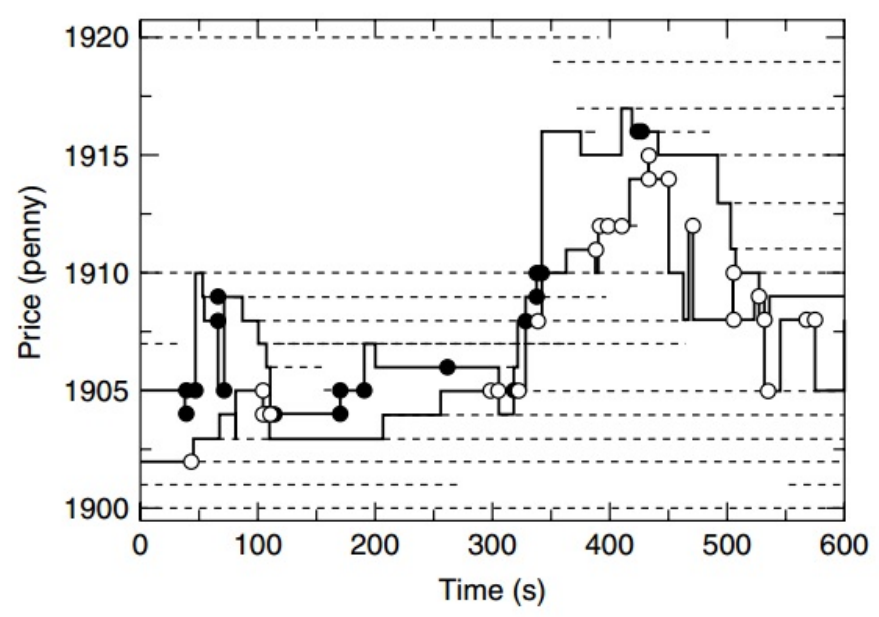

Figura 2.1: Representação gráfica da dinâmica do order book das ações de AstraZeneca negociadas na Bolsa de Londres, durante um intervalo de 10 min em 4 de setembro de 2002. Os círculos preenchidos(vazios) são operações de compra (venda). As linhas grossas são o preço bid e preço ask. As linhas tracejadas são limit orders.

inteiro do número mínimo permitido de ações em uma ordem, os tamanhos destas operações (volume de negociação) são tanto heterogêneos e fortemente auto-correlacionados. A unidade de discretização também pode variar ao longo do tempo e do mercado. A redução do tamanho do tick tem sido relacionada a vários fatos empíricos, como a diminuição do spread que é a diferença entre os preços ask e bid.

Estas mudanças de preços não são independentes: a função de autocorrelação dos retornos de preços é significativamente negativo no primeiro lag e depois rapidamente diminui para zero (Con01). Este é um típico efeito da microestrutura que desaparece quando se considera retornos em escalas de tempo mais longos.

\section{- Espaçamento temporal irregular}

Eventos como negociações, cotações e chegadas das ordens no order book não ocorrem em intervalos regulares de tempo, ao contrário eles ocorrem em intervalos de tempo espaçados irregularmente. Nesta estrutura o tempo entre os eventos é uma variável aleatória com correlação temporal, muitas vezes não triviais. A Figura 2.1 mostra um exemplo da ocorrência de espaçamento de tempo irregular de uma série de tempo financeira.

\section{- Durações correlacionadas}

As variações de preços ocorrem em intervalos de tempo irregulares e são chamadas durações, as durações entre variações de preços são aleatórias e endógenas, ou seja estão relacionados com o comportamento do preço e possivelmente com as durações anteriores. Em relação a modelos de séries temporais tradicionais, este propõe o problema adicional de modelar tais durações. Além disso as durações não são nem independentes nem exponencialmente distribuídos. A Figura 2.2 mostra um Q-Q plot comparando a distribuição das durações para o Citigroup com uma distribuição exponencial. As irregularidade de observações torna-se muito mais fácil de trabalhar com modelos de tempo continuo, em oposição aos modelos de séries temporais com base num intervalo de tempo fixo.

\section{- Periodicidade diária}

Séries financeiras de alta frequência mostram forte periodicidade diária. Das variáveis que têm uma periodicidade diária, podemos citar volatilidade, volume de negociações, número de transações, tempo entre negociações eo spread bid-ask. A periodicidade diária é devido ao 


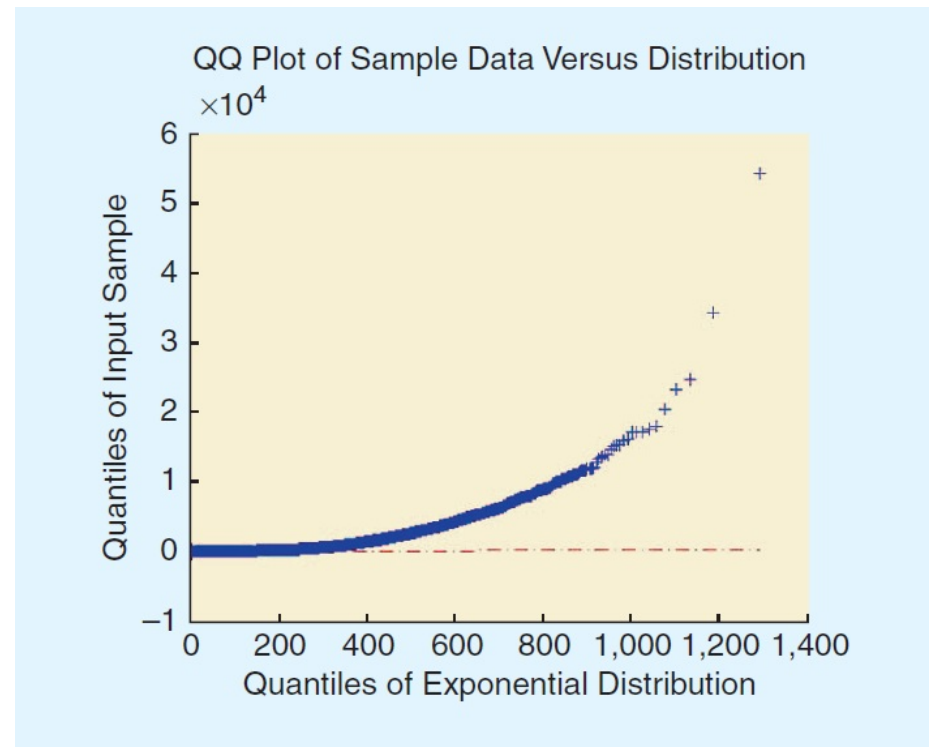

Figura 2.2: Quantis de durações comparados com quantis de uma distribuição exponencial com a mesma média (Citigroup, Junho de 2008). A linha tracejada representa os quantis de uma distribuição exponencial, que se encontram bastante diferentes dos quantis empíricos.

facto de a atividade de negociação não é constante ao longo do dia. Tipicamente, a atividade de negociação é elevada à volta da abertura, diminui até ao meio dia, e finalmente aumenta para o fim do dia de negociação. Isto é por vezes referido como um padrão em forma de $\mathbf{U}$ (conhecido como Smile). Um exemplo é mostrado na Figura 2.3. A Figura 2.3 (a)-(c) mostra o padrão intra-diário para o volume, a volatilidade, e número de operações para a General Electric na NYSE. Uma consequência importante da periodicidade diária é que as séries financeiras não são estacionárias porque as propriedades estatísticas das variáveis dependem da hora do dia. Além disso, a presença da forma em U produz periodicidades em as séries financeiras.

\section{- Correlações temporais}

Normalmente, as variáveis financeiras amostradas em alta frequência apresentam fortes correlações temporais. Algumas correlações temporais são específicas para microestrutura dos mercados financeiros em alta frequência. Outras correlações também estão presentes em uma frequência mais baixa, mas são mais intensas com dados intra-diários. Por exemplo, a Figura 2.4 mostra a função de autocorrelação dos retornos de preços de negociações para AstraZeneca (AZN) ações negociadas na LSE. A função de autocorrelação dos retornos dos preços de transacção é fortemente negativo no primeiro lag e depois rapidamente diminui para zero isso é muito bem conhecido como o pulo do spread bid-ask. Esta autocorrelação negativa desaparece quando se considera retornos agregados portanto é um efeito típico microestrutural. A Figura 2.4 mostra também a função de autocorrelação do valor absoluto dos retornos de preços de negociações, considerada aqui como uma medida de volatilidade. Esta função de autocorrelação decai muito lentamente indicando que a persistência eo agrupamento de volatilidade também pode ser observada nesta escala de tempo. Finalmente, muitas variáveis financeiras em alta frequência apresentam forte persistência temporal que muitas vezes podem ser modelado em termos de processos de memória longe. Exemplos incluem taxa de transação, spread, sinal do fluxo de ordens, e volume.

\section{- Estrutura do mercado}

Quando se trabalha com dados de alta frequência, é importante ter em conta a estrutura do mercado em estudo. Muitos mercados financeiros têm uma estrutura composta de múltiplos 

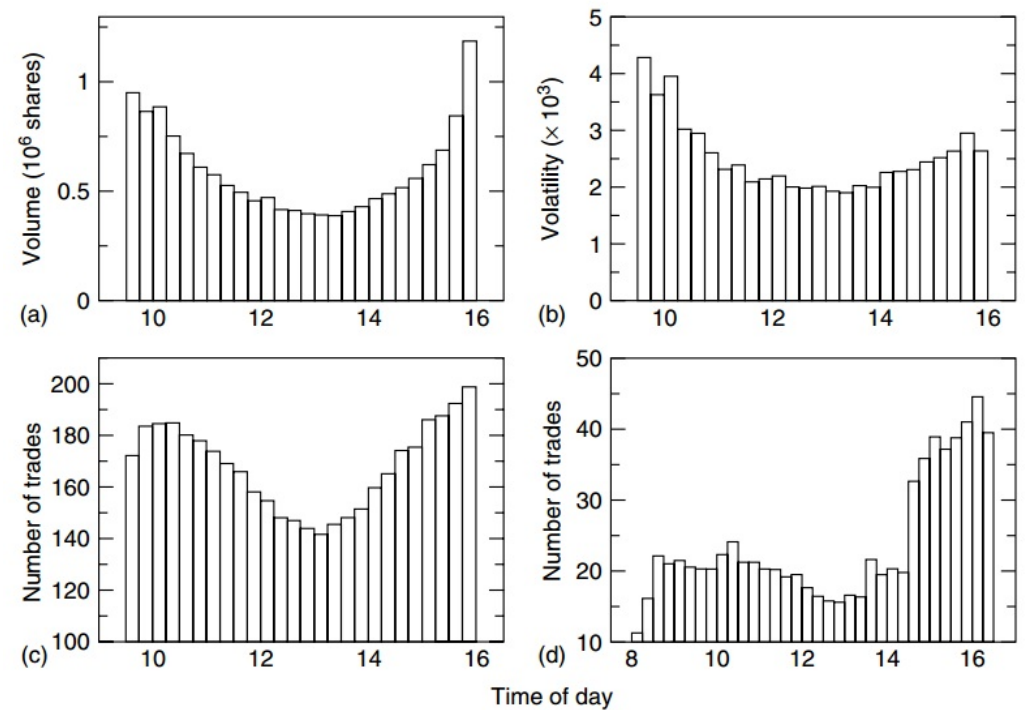

Figura 2.3: Exemplos de padrão intra-diário que registra 15 minutos de negociações eo eixo $x$ mostra a hora do dia. A figura mostra o volume negociado (a), variações de preço absoluto (b), eo número de negociações (c) da General Electric na NYSE durante o periodo de 2002-2003. (d) O número de negociações da AstraZeneca trocados no mercado eletrônico (SET1) do LSE durante o período de maio 2000 a dezembro de 2002

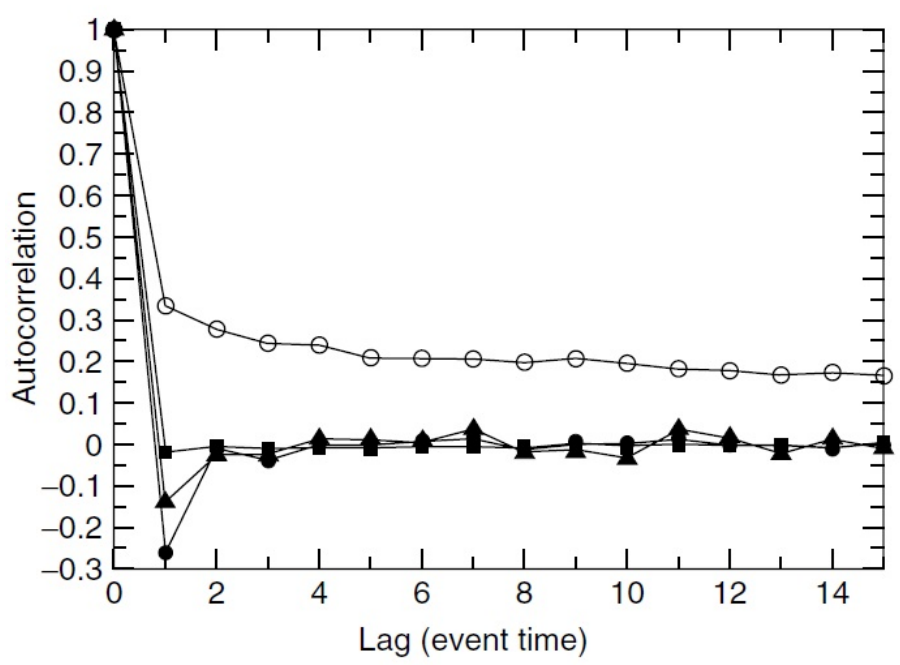

Figura 2.4: Função de autocorrelação dos retornos dos preços de transacção (círculos sólidos), valor absoluto dos retornos dos preços de transacção (círculos abertos), os retornos de preços médios amostradas antes de cada transação (triângulos a cheio), e retornos dos preços médios amostrados antes de cada evento de mercado (quadrados preenchidos) para AstraZeneca (AZN) ações negociadas na LSE em 2002. Os desfasamentos na linha horizontal são medidos em tempo do evento. 


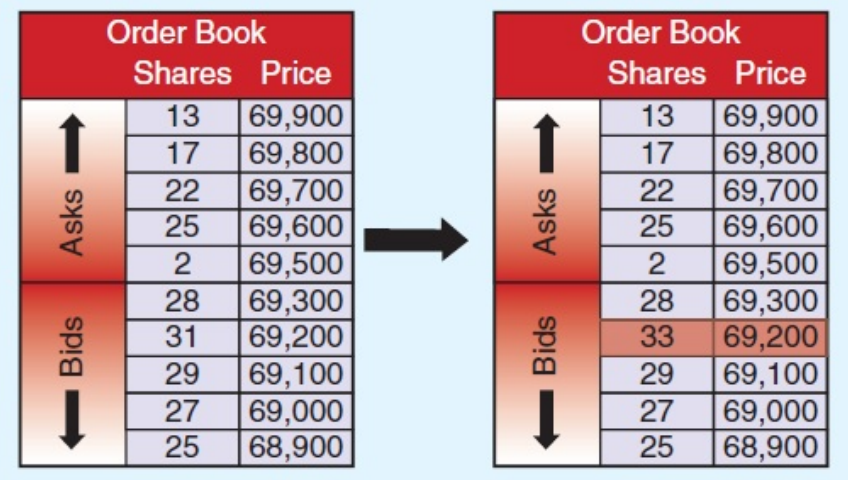

Figura 2.5: Um limit order de compra: comprar dois em 69.200.

\begin{tabular}{|c|c|c|c|c|c|}
\hline \multicolumn{3}{|c|}{ Order Book } & \multicolumn{3}{|c|}{ Order Book } \\
\hline \multirow{5}{*}{$\frac{\vec{m}}{\frac{9}{\alpha}}$} & 22 & 69,900 & \multirow{5}{*}{$\frac{\text { के }}{\frac{0}{2}}$} & 22 & 69,900 \\
\hline & 17 & 69,800 & & 17 & 69,800 \\
\hline & 140 & 69,700 & & 140 & 69,700 \\
\hline & 24 & 69,600 & & 24 & 69,600 \\
\hline & 6 & 69,500 & & 6 & 69,500 \\
\hline \multirow{5}{*}{ 음 } & 42 & 69,300 & \multirow{5}{*}{$\frac{0}{0}$} & 32 & 69,300 \\
\hline & 42 & 69,200 & & 42 & 69,200 \\
\hline & 41 & 69,100 & & 41 & 69,100 \\
\hline & 32 & 69,000 & & 32 & 69,000 \\
\hline & 21 & 68,900 & & 21 & 68,900 \\
\hline
\end{tabular}

Figura 2.6: Um market order de venda de 10.

mercados eletrônicos, mercados conexos, mercados em blocos, e assim por diante. As propriedades estatísticas de uma variável financeira podem ser bastante diferentes em mercados com diferentes estruturas. Portanto, na análise de dados de alta frequência, é importante ter em conta esta heterogeneidade na estrutura do mercado.

\subsection{Fluxo de ordens}

Os participantes do mercado podem enviar dois tipos de sinales numa ordem, de compra e venda. Um limit order é uma ordem para negociar uma certa quantidade de um título a um determinado preço chamado preço limite. Os limit orders são enviados para um sistema eletrônico de negociações, os estados dos limit orders pendentes enviados pelos participantes são resumidos indicando as quantidades enviadas afixadas em cada nível de preço e com seu sinal correspondente: esse registro é conhecido como o limit order book. Um exemplo de um limit order book é mostrado na Figura 2.5. O preço mais baixo para o qual existe um limit order de venda pendente é chamado o preço ask eo preço de compra mais alto é chamado o preço bid.

Um market order é uma ordem para comprar ou vender uma certa quantidade do ativo com o melhor preço disponível no limit order book. Quando um market order chega, ele é combinado com um limit order como o melhor preço disponível no limit order book ea negociação ocorre automaticamente. As quantidades disponíveis no bid/ask no limit order book são reduzidos em $x$ quando um market order de tamanho $x$ é executada. A Figura 2.6 mostra a evolução de um limit order book quando um market order é executada.

Um limit order fica no order book, até que o mesmo seja executado contra um market order ou ate que seja cancelado. Um limit order pode ser executado muito rapidamente se ele corresponde a 
um preço próximo do preço bid/ask, mas pode levar um longo tempo se o preço se afasta demasiado longe do preço bid/ask. Como alternativa, um limit order pode ser cancelado a qualquer momento. $\mathrm{Na}$ maioria dos mercados eletrônicos, a maioria dos limit order são cancelados pouco depois de ser enviados: para muitas ações líquidas da NYSE e NASDAQ, até $80 \%$ dos limit order são canceladas em menos de um segundo de ser enviados.

Os fluxos de ordens é o processo conjunto de colocações e cancelamentos de market e limit orders. O processo de formação de preços resulta da interação entre esses eventos na qual a seu vez é o foco da teoria de microestrutura de mercado. O Fluxo de ordens está relacionada com a oferta, demanda e com a liquidez, o fluxo de limit orders aumenta a liquidez do ativo, enquanto os fluxos de market orders e cancelamentos esgotam a liquidez do ativo.

\subsubsection{Propriedades estatísticas dos fluxos de ordens}

O fluxo de ordens é um processo estocástico complexo que ocorre em tempo contínuo. Ele tem três componentes (limit orders, market orders e cancelamentos), cada componente sendo caracterizado por um volume, um preço e uma sinal. O fluxo de ordens é, além disso, fortemente dependente do estado atual do limit order book. Não há um modelo estocástico disponível capaz de descrever completamente a estrutura de dependência deste processo. O que normalmente é feito é restringe a uma sub-parte do fluxo de ordens e descrevê-lo dentro de um modelo reduzido adequado.

A fim de simplificar a apresentação, neste parte do trabalho centra-se na modelagem estatística de cada componente separadamente.

\section{- Fluxo de market orders}

A função de densidade do volume de market orders é frequentemente descrita por uma função decrescente, as caudas tem decrescimento polinomial (ver figura 2.7) . O fluxo de market orders tem interessantes propriedades de correlações temporais. Considere, por simplicidade, a série temporal simbólica obtida na hora do evento, substituindo os market orders de compra com +1 e os market orders de venda com -1 , independentemente do volume da ordem. A função de autocorrelação $C(\tau)$ dessas sequências de ordens decai muito lentamente para zero e seu comportamento assintótico é ajustado muito bem por uma função polinomial

$$
C(\tau) \approx \frac{1}{\tau^{\alpha}}
$$

Donde $\alpha<1$ (tipicamente $\alpha \cong 0.5$ ) [15]. Processos com tais autocorrelações são chamados processos de memoria longe. O painel (a) da figura 2.8 mostra a função de autocorrelação dos market orders para AstraZeneca (AZN) negociados na LSE. Vale a pena notar que a persistência no fluxo de market orders ainda é estatisticamente significativa após muitas horas de negociação. O fluxo de market orders ponderado pelo volume mostra forte persistência temporal a pesar de que é enfraquecida pelas flutuações de volume (veja a Figura 2.8 b).

As propriedades condicionais de fluxo de market orders também são importantes. Primeiro, o tamanho do spread bid-ask é um fator determinante do fluxo de market orders. A taxa de market orders, independente seja de compra ou venda, diminui à medida que aumenta $o$ spread (ver Figura 2.9). Esse comportamento é intuitivamente esperado já que um grande spread bid-ask é um forte desincentivo à negociação, dado que o custo relacionado é grande. Uma segunda propriedade condicional diz respeito ao volume de uma ordem no mercado, o que é fortemente dependente do volume com o melhor preço oposto. Uma segunda propriedade condicional diz respeito ao volume de um market order, o que é fortemente dependente do volume do melhor preço oposto. Especificamente, é raro que o volume de um market order seja superior ao volume do melhor oposto. Isso indica que os agentes condicionam o tamanho de suas market order à liquidez existente no mercado, fazendo grandes transações somente quando a liquidez é alta e pequenas quando é baixa. 


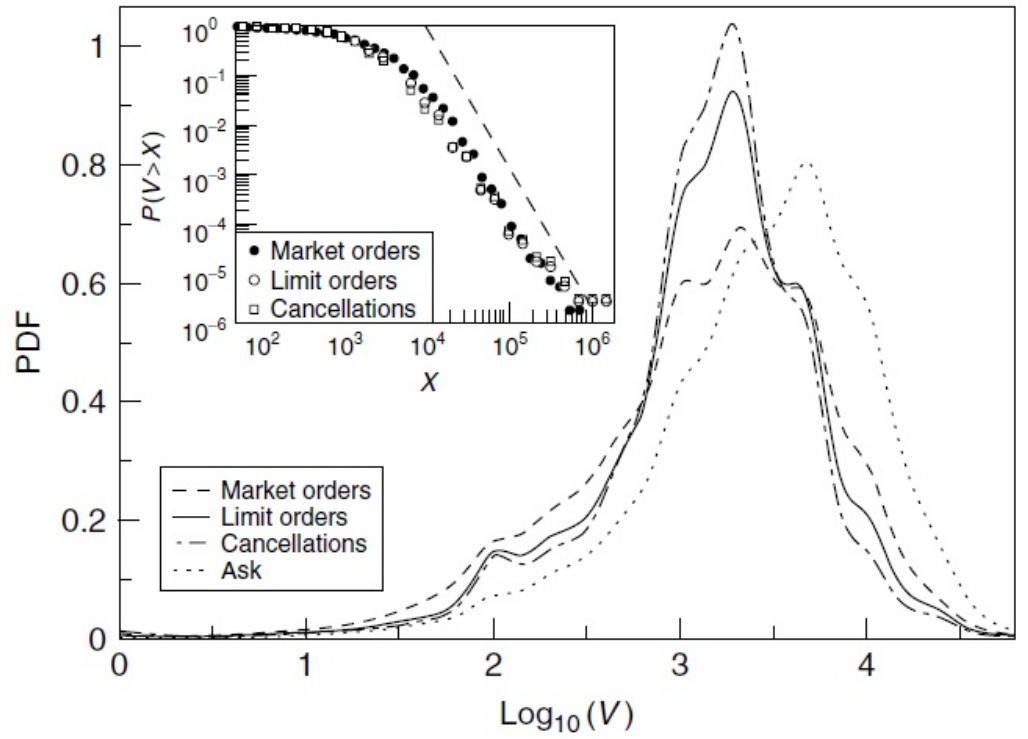

Figura 2.7: Função densidade de probabilidade do log-volume de ordens individuais, para os três componentes diferentes do fluxo de ordens. Para efeito de comparação, a figura também mostra a função densidade de probabilidade de logaritmo decimal do volume no melhor preço (ask), amostrados antes de cada transação. A inserção mostra em uma escala logarítmica dupla a distribuição de probabilidade acumulada do volume para os diferentes tipos de ordnes. A linha pontilhada é uma função polinomial com inclinação de 2,8, que é o estimador de máxima verossimilhança do expoente de cauda para os order market. Os dados referem-se ao AZN ações negociadas na LSE no período 1999-2002.
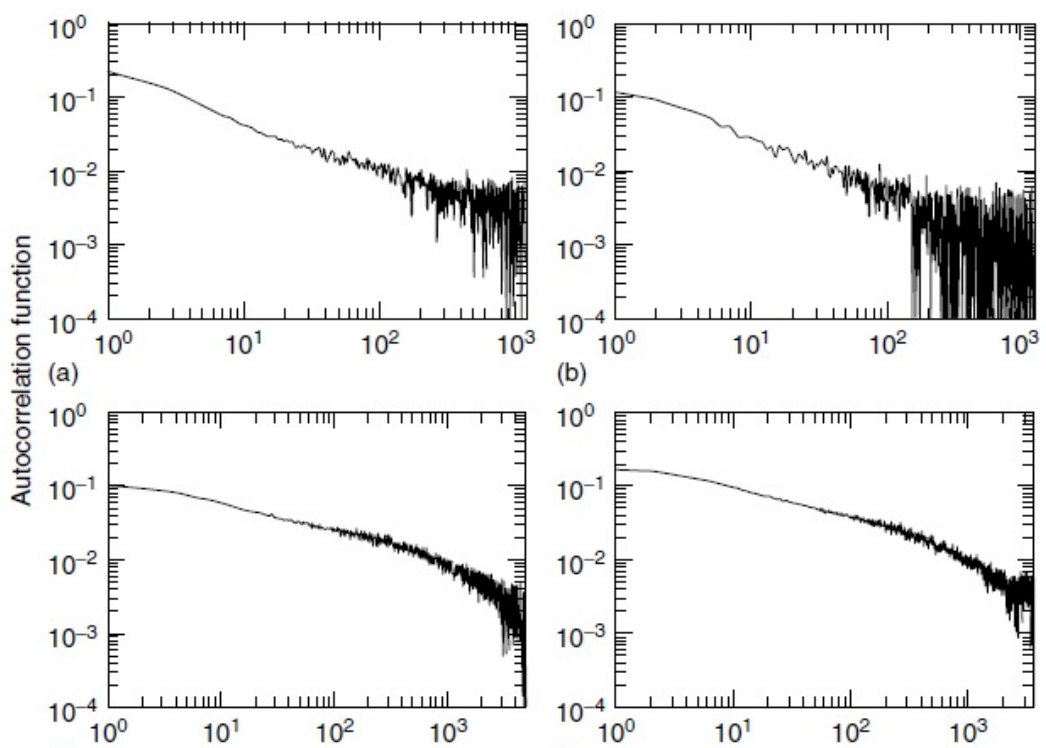

(b)

(c)

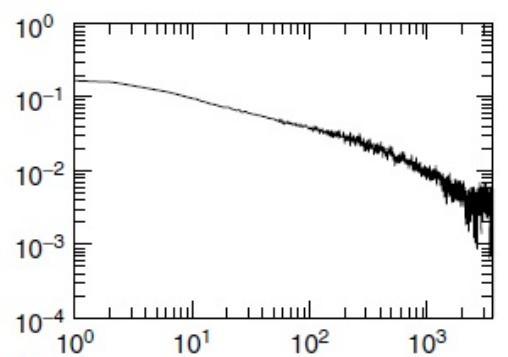

(d)

Lag (number of events)

Figura 2.8: Autocorrelation dos fluxos de ordens plotados em uma escala logarítmica dupla. Especificamente, os painéis mostram a função de autocorrelação do market order (a), volume dos market orders (b), limit orders (c), e cancelamentos (d). Os dados referem-se ao AZN ações negociadas na LSE no período 1999-2002 


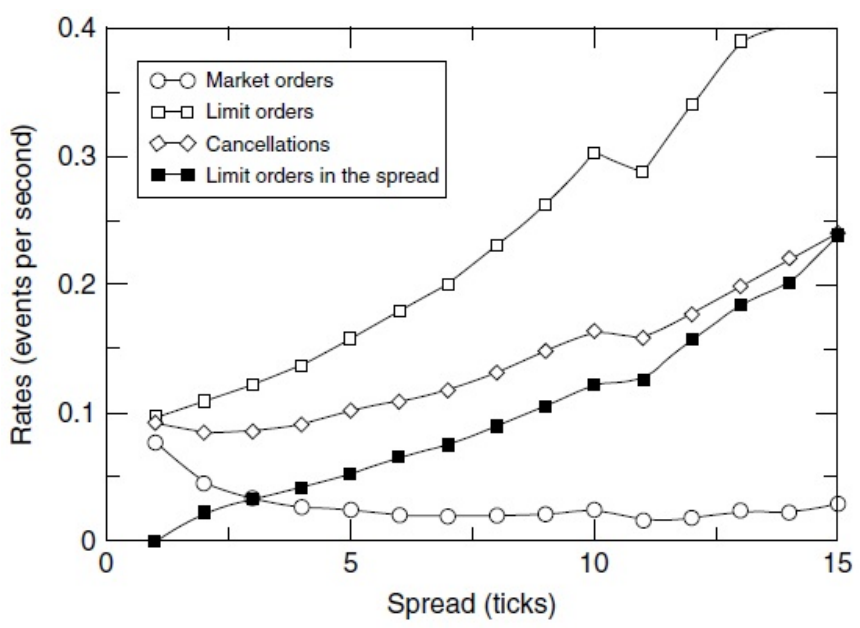

Figura 2.9: Taxas de diferentes tipos de eventos no limit order book condicionados ao tamanho do spread. Os dados referem-se a ações de AZN negociados na LSE em 2002.

\section{- Fluxo de limit orders}

Um limit order é caracterizada pelo volume e seu preço limite. Várias regularidades estatísticas do limit order também foram observados. Em primeiro lugar, a distribuição incondicional de volume do limit order é semelhante ao de market order (ver Figura 2.7). Em segundo lugar, a série temporal dos limit order também são caracterizado pela propriedade de memoria longe. A Figura 2.8 (c) mostra a função de autocorrelação dos limit order para AZN. Em terceiro lugar, diversas regularidades estatísticas do fluxo de limit orders são observadas quando se considera também o preço limite. O preço limite caracteriza a agressividade do limit order ea paciência do operador desde que a probabilidade de que a ordem seja executada em um determinado intervalo de tempo depende de onde ele é colocado. Limit orders no spread são muito agressivos e mais propensos a ser executado, enquanto os limit orders colocados no order book muito longe do melhor preço são menos agressivos e sua probabilidade de execução é baixa. Para ações de alta liquidez na LSE entre 1999 e 2002, cerca de um terço dos limit order foram colocados no interior do spread, outro terço com os melhores preços, eo último terço dentro do order book. Uma das regularidades estatísticas observadas recentemente é a distribuição polinomial do preço dos limit orders nos mercados financeiros de leilão duplo contínuas. Seja $b(t)-\triangle$ denota o preço de um novo limit order de compra e $a(t)+\triangle$ o preço de um novo limit order de venda. Daqui $a(t)$ é o preço ask e $b(t)$ é o preço bid, quando um limit order é colocado. A função de densidade $\rho(\triangle)$ do preço relativo $\triangle$ é muito semelhante para ordens de compra e venda. Além disso, para grandes valores de $\triangle$ a função de densidade de probabilidade tem um bom ajuste a uma função polinomial

$$
\rho(\triangle) \approx \frac{1}{\triangle^{1+\mu}}
$$

na qual $\mu \approx 1.5$ para as ações da LSE, e $\mu \approx 0.6$ para as ações negociadas na Bolsa de Paris (BMP02).

A taxa de colocação de limit orders depende fortemente do spread. Quando o spread é grande, a taxa de colocação de limir orders também é grande (veja a Figura 2.9p. Isso também é verdade quando se considera só os limit orders colocados no interior do spread. A razão é que um spreas grande é um incentivo para fornecer liquidez e para adquirir prioridade de preço colocando um limit order no spread. Então o fluxo de limit orders depende em grande medida do estado atual do order book. 


\section{- Fluxo de cancelamentos}

Um limit order pode ser cancelado ou pode expirar, ambos eventos levam a um esgotamento de liquidez. Além disso, o volume de limit orders cancelados é distribuído de forma semelhante ao volume de limit orders (ver Figura 2.7). A série temporal de cancelamento também exibe a propriedade memoria longe (ver Figura 2.8 d). Além disso, a taxa de cancelamentos aumenta com o tamanho do spread (ver Figura 2.9). Isto é provavelmente devido à alta atividade simultânea de colocação e cancelamentos de limit orders quando uma crise de liquidez temporária provoca uma a competição entre fornecedores de liquidez a fim de encontrar um novo valor de equilíbrio para os preços bid e aks. Finalmente, o tempo de vida de um determinado limit order (que é inversamente proporcional à probabilidade de cancelamento) aumenta à medida que se afasta do spread bid-ask (BMP02). Esse fenômeno pode ser explicado considerando que as ordens distantes são normalmente colocadas no mercado por investidores pacientes que pretendam-se beneficiar de oscilações significativas nos preços a médio prazo, enquanto as ordens perto dos preços bid e aks correspondem aos participantes de mercado muito ativos que reajustam suas ordens, com uma frequência muito alta. A probabilidade de cancelamento no tempo $t$ depende também da razão entre a distancia $\Delta$ do preço limite ao melhor preço no tempo $t$ e no tempo $t_{0}<t$ quando a ordem foi colocada. Mike e Farmer ajustam a probabilidade de cancelamento a uma forma funcional $\left(\overline{\mathrm{FGL}^{+} 04}\right)$.

$$
p(t) \alpha 1-\exp \left[-\frac{\triangle(t)}{\triangle\left(t_{0}\right)}\right]
$$

o que significa que, se o preço se move em uma direção oposta ao limit order, a probabilidade de cancelamento cresce. 


\section{Capítulo 3}

\section{Modelo estocástico para a dinâmica de dados order book}

\subsection{Introdução}

Neste Capítulo mostramos um modelo estocástico em tempo contínuo para a dinâmica do limit order book, proposto por Cont, Stoikov e Talreja (CST10). O modelo estabelece um equilíbrio entre duas características que são desejáveis em um modelo para dados de alta frequência: tenta capturar as propriedades estatísticas e fatos empíricos fundamentais da dinâmica do order book discutidas no Capítulo 2, e obtém resultados semianalíticos que permitem cálculos rápidos de diversas quantidades de interesse, sem recorrer a simulação. Usando métodos de transformada de Laplace, o modelo é capaz de calcular de forma eficiente probabilidades de vários eventos, condicionados a estados atuais do order book e captura a dinâmica de curto prazo com boa precisão.

\subsection{Descrição do modelo}

Modelamos a dinâmica do order book como um processo de Markov de tempo contínuo. Na qual $\{1, \ldots, n\}$ representam todos os níveis de preços permitidos que são múltiplos de um tick, o limite superior $n$ é escolhido suficientemente grande de modo que ordens com preços mais elevados são altamente improváveis no período de nossa análise, uma vez que o modelo destina-se a ser utilizado em escalas de tempo de corto prazo.

Define $\{X(t)\}_{t>0}=\left(X_{1}(t), \ldots X_{n}(t)\right)_{t>0}$ a cadeia de Markov em tempo continuo, e $\mathbb{Z}^{n}$ como espaço de estados, onde $\left|X_{p}(t)\right|$ é o numero de limit order pendentes no preço $p, 1 \leq p \leq n$. Se $X_{p}(t)<0$, então existem $-X_{p}(t)$ ordens bid (ordens de compra) ao preço $p$, se $X_{p}(t)>0$ então existem $X_{p}(t)$ ordens ask (ordens de venda) ao preço $p$.

Antes de prosseguir descrevendo o modelo definimos:

Definição 3.2.1. O preço ask é o preço de venda mais baixo.

$$
p_{A}(t)=\inf \left\{i: X_{i}(t)>0\right\}
$$

Definição 3.2.2. O preço bid é o maior preço de compra..

$$
p_{B}(t)=\sup \left\{i: X_{i}(t)<0\right\}
$$

Definição 3.2.3. O preço médio eo spread bid-ask são definidos como:

$$
p_{M}(t)=\frac{p_{B}(t)+p_{A}(t)}{2}
$$




$$
p_{S}(t)=p_{A}(t)-p_{B}(t)
$$

A chegada de uma nova ordem é representado por uma transição na cadeia de Markov, enquanto no mercado o tamanho das ordens mudam, neste modelo, o tamanho das ordens é fixo em una unidade, este tamanho pode ser escolhido como o tamanho médio de todas as ordens.

\subsection{Suposições do modelo}

- Os maket order de compra (resp. venda) chegam independente e com tempos exponenciais com taxa $\mu$.

- Os limit order de compra (resp. venda) chegam a uma distância de $i$ ticks da melhor cotação oposto com tempos exponenciais, independentes e com taxa $\lambda(i)$.

- Os cancelamentos dos limit order a uma distância de $i$ ticks da melhor cotação oposto ocorrer a uma taxa proporcional ao número de ordens pendentes: se o número de ordens pendentes nesse nível é $x$ então a taxa de cancelamento é de $\theta(i) x$.

- Os eventos acima são independentes entre si.

- Assumir que todas as ordens são do tamanho da unidade (em exemplos empíricos vamos tomar como unidade ao tamanho médio das ordens).

\subsection{Dinâmica do order book}

Para um estado $x \in \mathbb{Z}^{n}$ e $1 \leq p \leq n$ definimos $x+e_{p}=x+(0, \ldots, 1, \ldots, 0)$ e denote a transição de um estado $x \in \mathbb{Z}^{n}$ para $x+e_{p}$ as $x \rightarrow x+e_{p}$.

1. um limit order de compra no preço $p<p_{A}(t)$ aumenta a quantidade do nível $p$ em uma unidade

$$
x \rightarrow x-e_{p} \quad \text { com taxa } \quad \lambda\left(p_{A}(t)-p\right) \quad \text { para } \quad p<p_{A}(t)
$$

2. um limit order de venda no preço $p>p_{B}(t)$ aumenta a quantidade do nível $p$ em una unidade

$$
x \rightarrow x+e_{p} \quad \text { com taxa } \quad \lambda\left(p-p_{B}(t)\right) \quad \text { para } \quad p>p_{B}(t)
$$

3. um market order de compra diminui a quantidade no preço ask em uma unidade

$$
x \rightarrow x+e_{p_{B}(t)} \quad \text { com taxa } \quad \mu
$$

4. um market order de venda diminui a quantidade no preço bid em uma unidade

$$
x \rightarrow x-e_{p_{A}(t)} \quad \text { com taxa } \quad \mu
$$

5. um cancellation order de um limit order de venda pendente diminui a quantidade do nível $p$ em uma unidade

$$
x \rightarrow x-e_{p} \quad \text { com taxa } \quad \theta\left(p-p_{B}(t)\right)\left|x_{p}\right| \text { para } \quad p>p_{B}(t)
$$

6. um cancellation order de um limit order de compra pendente diminui a quantidade do nível $p$ em uma unidade

$$
x \rightarrow x+e_{p} \quad \text { com taxa } \quad \theta\left(p_{A}(t)-p\right)\left|x_{p}\right| \quad \text { para } \quad p<p_{A}(t)
$$


Definição 3.4.1. Dizemos que um estado é admissivel se:

$$
\begin{aligned}
& \mathbb{A}=\left\{x \in \mathbb{Z}^{n}: \exists k, l \in \mathbb{N}, 1 \leq k<l \leq n, x_{p} \leq 0 \quad \text { for } \quad p \leq k\right. \\
& x_{p}=0 \text { for } k<p<l \\
& \left.x_{p} \geq 0 \text { for } p \geq l\right\}
\end{aligned}
$$

Se o estado inicial do order book é admissível, continua a ser admissível com probabilidade um:

Proposição 3.4.1. Se $X(0) \in \mathbb{A}$ então $P(X(t) \in \mathbb{A})=1$.

É facilmente verificável que $\mathbb{A}$ é estável em cada um dos seis transições definidas acima, o que nos leva a nossa afirmação.

Proposição 3.4.2. Se $\theta=\min _{1 \leq i \leq n} \theta(i)>0, X$ é um processo de Markov ergodico.

Demonstração. Pelo Teorema 1.4 de (MP12), Uma cadeia irredutível e aperiódica é recorrente positiva se existe uma função $f$ positiva, que tende ao infinito (chamada função de Lyapunov), uma constante $\epsilon>0$ e um conjunto finito $F$ conteúdo no espaço de estados; de tal modo que $\Gamma f(x) \leq-\epsilon$ para todo $x \notin F$, onde $\Gamma$ é o gerador da cadeia de Markov $X(t)$.

Para um estado $x \in \mathbb{Z}^{n}$ o gerador da cadeia aplicado a uma função é dado por:

$$
\begin{gathered}
\Gamma f(x)=\sum_{p<p_{A}} f\left(x-e_{p}\right) \lambda\left(p_{A}-p\right)+\sum_{p>p_{B}} f\left(x+e_{p}\right) \lambda\left(p-p_{B}\right)+f\left(x+e_{p_{B}}\right) \mu+f\left(x-e_{p_{A}}\right) \mu+ \\
\sum_{p<p_{A}} f\left(x+e_{p}\right) \theta\left(p_{A}-p\right)\left|x_{p}\right|+\sum_{p>p_{B}} f\left(x-e_{p}\right) \theta\left(p-p_{B}\right)\left|x_{p}\right|
\end{gathered}
$$

Consideramos como função de Lyapunov o número total de ordens no order book em estado determinado, em outras palavras $f(x)=\sum_{i=1}^{n}\left|x_{i}\right|$, notar que $f \uparrow \infty$. O gerador aplicado nessa função é dado por

$$
\Gamma f(x)=\sum_{p<p_{A}} \lambda\left(p_{A}-p\right)+\sum_{p>p_{B}} \lambda\left(p-p_{B}\right)-2 \mu-\sum_{p<p_{A}} \theta\left(p_{A}-p\right)\left|x_{p}\right|-\sum_{p>p_{B}} \theta\left(p-p_{B}\right)\left|x_{p}\right|
$$

Então a seguente desigualdade é valida para todo elemento do espaço de estados, seja $x \in \mathbb{Z}^{n}$

$$
\Gamma f(x) \leq \lambda-2 \mu-\theta \sum_{1 \leq i \leq n}\left|x_{i}\right| \quad \text { na qual } \quad \lambda=\sum_{1 \leq i \leq n} \lambda(i)
$$

Dado que $\lambda, \mu$ e $\theta>0$ são quantidades finitas, e dado que $\sum_{1 \leq i \leq n}\left|x_{i}\right|$ diverge conforme $n$ cresce, existirá sempre um $N$ tal que para todo $n \geq N$, acontece que: $2 \mu+\theta \sum_{1 \leq i \leq n}\left|x_{i}\right|>\lambda$.

Então a partir disso podemos concluir que $\Gamma f(x) \leq-\epsilon$ para algum $\epsilon>0$ para todo $x \notin F$ onde $F=\left\{x \in \mathbb{Z}^{n}: 2 \mu+\theta \sum_{1<i<n}\left|x_{i}\right|<\lambda\right\}$, isso demonstra que $X(t)$ é recorrente positivo. Desde que $X(t)$ é claramente irredutível e aperiódico, resulta que $X(t)$ é ergódico.

\subsection{Transformadas de Laplace para calcular probabilidades condi- cionais}

\subsubsection{Tempos de primeira passagem de processos nascimento-morte}

A fracção contínua associada com a sequência $\left\{a_{n}\right\}_{n \in \mathbb{N}}$ como numeradores parciais e $\left\{b_{n}\right\}_{n \in \mathbb{N}}$ como denominadores parciais, que são números complexos com $a_{n} \neq 0$ para todo $n \in \mathbb{N}$, é a sequência $\left\{w_{n}\right\}_{n \in \mathbb{N}}$ gerado por 


$$
w_{n}=t_{1} \circ t_{2} \circ \ldots \circ t_{n}(0)
$$

onde

$$
t_{k}(u)=\frac{a_{k}}{b_{k}+u}
$$

Se $\lim _{n \rightarrow \infty} w_{n}=w$, então a fracção contínua é dito convergente e o limite $w$ é o valor da fracção contínua. Neste caso, escrevemos

$$
w=\Phi_{n=1}^{\infty} \frac{a_{n}}{b_{n}}
$$

Considere agora um processo de nascimento-morte com taxa de nascimento constante $\lambda$ e as taxas de morte $\mu_{i}$ no estado $i \geq 1$, e denote como $\sigma_{b}$ ao tempo de primeira passagem a 0 dado que começo no estado $b$. Observe também podemos escrever $\sigma_{b}$ como a soma

$$
\sigma_{b}=\sigma_{b, b-1}+\sigma_{b-1, b-2}+\ldots+\sigma_{1,0}
$$

onde $\sigma_{i, i-1}$ denota o tempo de primeira passagem do processo nascimento-morte do estado $i$ para o estado $i-1$, para $i=1, \ldots, b$, e todos os termos no lado direito são independentes. Se $\hat{f}_{b}$ denota a transformada de Laplace de $\sigma_{b}$ e $\hat{f}_{i, i-1}$ denota a transformada de Laplace de $\sigma_{i, i-1}$, para $i=1, \ldots, b$, então temos

$$
\hat{f}_{b}(s)=\prod_{i=1}^{b} \hat{f}_{i, i-1}(s) .
$$

Pela Equação (4.9) de (AW99), vemos que a transformada de Laplace $\hat{f}_{i, i-1}(s)$ é dado por

$$
\hat{f}_{i, i-1}(s)=-\frac{1}{\lambda} \Phi_{k=i}^{\infty} \frac{-\lambda \mu_{k}}{\lambda+\mu_{k}+s} .
$$

para $i=1, \ldots, b$.

Combinando 3.5 .1 e 3.5 .2 obtemos

$$
\hat{f}_{b}(s)=\left(-\frac{1}{\lambda}\right)^{b}\left(\prod_{i=1}^{b} \Phi_{k=i}^{\infty} \frac{-\lambda \mu_{k}}{\lambda+\mu_{k}+s}\right) .
$$

Usaremos este resultado em todos nossos cálculos abaixo.

\subsubsection{Direção de movimentos de preços}

Nós agora calculamos a probabilidade de aumento do preço médio no próximo movimento, dado um estado atual do order book. Desde que o preço meio é dado por $p_{M}(t)=p_{A}(t)+p_{B}(t) / 2$, o preço meio muda, se somente se, o preço bid ou o preço ask mudam. Nós analisamos o movimento de preços em dois casos diferentes

1. Se o spread $S=1$ então o primeiro movimento do preço médio ocorre quando a fila de ordens do preço ask $\left(X_{p_{A}}\right)$ ou preço bid $\left(X_{p_{B}}\right)$ atingem zero. Se a primeira fila em atingir zero é $X_{p_{A}}$ então o preço médio subira, por outro lado, se $X_{p_{B}}$ é o primeiro em atingir zero o preço médio cai.

2. Se o spread $S>1$ então o primeiro movimento do preço médio ocorre quando uma das filas $X_{p_{B}}, X_{p_{B}}$ atingem zero, como foi dito antes, quando um limit order é colocado entre $p_{B}$ e $p_{A}$, se o limit order colocado no spread é um ordem bid o preço médio subiria, se é uma ordem ask o preço médio cairia. 
Seja $T$ o tempo da primeira mudança no preço médio:

$$
T=\inf \left\{t \geq 0: p_{M}(t) \neq p_{M}(0)\right\}
$$

e denote

$$
\left\{\begin{array}{c}
X_{p_{A}(\cdot)}(\cdot)=X_{A} \\
\left|X_{p_{B}(\cdot)}(\cdot)\right|=X_{B}
\end{array}\right.
$$

Dado o estado atual do order book, a probabilidade de um aumento do preço médio na próxima mudança é

$$
P\left(p_{M}(T)>p_{M}(0) \mid X_{A}=a, X_{B}=b, p_{S}(0)=S\right)
$$

Lema 3.5.1. Existem processos independentes de nascimento-morte $\tilde{X}_{A}$ e $\tilde{X}_{B}$. com taxas de nascimento constantes $\lambda(S)$ e taxas de morte $\mu+i \theta(S)$, de modo que para todo $0 \leq t \leq T, \tilde{X}_{A}(t)=X_{A}(t)$ e $\tilde{X}_{B}(t)=X_{B}(t)$.

Demonstração. Nosso modelo é dado por $\{X(t)\}_{t>0}=\left(X_{1}(t), \ldots X_{n}(t)\right)_{t>0}$, cada coordenada $X_{i}$ não constitui em um processo de nascimento-morte. No entanto, este lema diz que enquanto o preço médio não muda, $X_{i}$ se comporta como um processo de nascimento-morte. Para $0 \leq t<T$ nós temos $p_{A}(t)=p_{A}(0)$ e $p_{B}(t)=p_{B}(0)$, então $X_{A}(t)$ e $X_{B}(t)$ têm as seguintes taxas de transição

$$
\begin{array}{lll}
n \rightarrow n+1 & \text { com taxa } & \lambda(S) \\
n \rightarrow n-1 & \text { com taxa } & \mu+n \theta(S)
\end{array}
$$

Define $\tilde{X}_{A}(t)=X_{A}(t)$ e $\tilde{X}_{B}(t)=X_{B}(t)$ para $t \leq T$. e $\tilde{X}_{A}(t), \tilde{X}_{B}(t)$ seguem com as mesmas taxas de transição para $t>T$.

Teorema 3.5.2. Seja $\hat{f}_{j}^{s}$

$$
\hat{f}_{j}^{S}(s)=\left(-\frac{1}{\lambda(S)}\right)^{j}\left(\prod_{i=1}^{b} \Phi_{k=1}^{\infty} \frac{-\lambda(S)(\mu+k \theta(S))}{\lambda(S)+\mu+k \theta(S)+s}\right)
$$

quando $S=1$ a probabilidade 3.5.4 é dado pela transformada inversa de Laplace de

$$
\hat{F}_{\sigma_{A}-\sigma_{B}}(s)=\frac{1}{s} \hat{f}_{a}^{1}(s) \hat{f}_{b}^{1}(-s)
$$

avaliada em 0.

Demonstração. Primeiro construir o processo de nascimento-morte $\tilde{X}_{A}$ e $\tilde{X}_{B}$ tal como no lema anterior e, respectivamente, seja $\sigma_{A}$ e $\sigma_{B}$ ser o tempo de primeira passagem a zero . O preço médio subira se a primeira fila para chegar a zero é $X_{p_{A}}$ ou em outras palavras $\sigma_{A}<\sigma_{B}$. A probabilidade (3.5.4) é dado por

$$
P\left(\sigma_{A}<\sigma_{B} \mid X_{A}=a, X_{B}=b\right)=P\left(\sigma_{A}-\sigma_{B}<0 \mid X_{A}=a, X_{B}=b\right)=F_{\sigma_{A}-\sigma_{B}}(0)
$$

mas

$$
\hat{F}_{\sigma_{A}-\sigma_{B}}(s)=\frac{1}{s} \hat{f}_{\sigma_{A}-\sigma_{B}}^{1}(s)=\frac{1}{s} \hat{f}_{a}^{1}(s) \hat{f}_{b}^{1}(-s)
$$

Assim, a probabilidade desejada é obtida a partir da transformada inversa de Laplace de 3.5.8 avaliada em 0 .

Lema 3.5.3. Seja $Z$ uma variável aleatória distribuído exponencialmente com parâmetro $\Lambda$. Então, a transformada de Laplace da variável aleatória $\min \left\{\sigma_{B}, Z\right\}=\sigma_{B} \wedge Z$ é dado por:

$$
\hat{f}_{\sigma_{B} \wedge Z}=\hat{f}_{b}^{1}(\Lambda+s)+\frac{\Lambda}{\Lambda+s}\left(1-\hat{f}_{b}^{1}(\Lambda+s)\right)
$$


Demonstração. Primeiro calculamos a função de distribuição de $\sigma_{B} \wedge Z$.

$$
\begin{aligned}
F_{\sigma_{B} \wedge Z}(t)=P\left(\sigma_{B} \wedge Z<t\right) & =1-P\left(\sigma_{B} \wedge Z \geq t\right) \\
& =1-P\left(\sigma_{B} \geq t\right) P(Z \geq t) \\
& =1-\left(1-F_{\sigma_{B}}(t)\right) e^{-\Lambda t} \\
& =F_{\sigma_{B}}(t) e^{-\Lambda t}
\end{aligned}
$$

Tomando as derivadas em relação a $t$ dá

$$
f_{\sigma_{B} \wedge Z}(t)=f_{b}^{1}(t) e^{\Lambda t}+\Lambda\left(1-F_{\sigma_{B}}(t)\right) e^{-\Lambda t}
$$

A transformada de Laplace de $\sigma_{B} \wedge Z$ é dado por

$$
\begin{aligned}
\hat{f}_{\sigma_{B} \wedge Z}(s) & =\int_{0}^{\infty} e^{-s t} f_{\sigma_{B} \wedge Z}(t) d t \\
& =\int_{0}^{\infty} e^{-s t}\left(f_{b}^{1}(t) e^{\Lambda t}+\Lambda\left(1-F_{\sigma_{B}}(t)\right) e^{-\Lambda t}\right) d t \\
& =\int_{0}^{\infty} e^{-(\Lambda+s) t} f_{b}^{1}(t) d t+\Lambda \int_{0}^{\infty}\left(1-F_{\sigma_{B}}(t)\right) e^{-(\Lambda+s) t} d t \\
& =\hat{f}_{b}^{1}(\Lambda+s)+\frac{\Lambda}{\Lambda+s}\left(1-\hat{f}_{b}^{1}(\Lambda+s)\right)
\end{aligned}
$$

Teorema 3.5.4. Quando $S>1$ a probabilidade (3.5.4) é dado por a transformada inversa de Laplace de

$$
\begin{aligned}
& \hat{F}^{S}=\frac{1}{s}\left[\hat{f}_{a}^{S}\left(\Lambda_{S}+s\right)+\frac{\Lambda_{S}}{\Lambda_{S}+s}\left(1-\hat{f}_{a}^{S}\left(\Lambda_{S}+s\right)\right)\right]\left[\hat{f}_{b}^{S}\left(\Lambda_{S}+s\right)+\frac{\Lambda_{S}}{\Lambda_{S}+s}\left(1-\hat{f}_{b}^{S}\left(\Lambda_{S}+s\right)\right)\right] \\
& \text { na qual } \hat{f}_{b}^{S} \text { é definida no teorema } 3.5 .2 \text { e } \Lambda_{S}=\sum_{i=1}^{S-1} \lambda(i)
\end{aligned}
$$

\subsection{Comportamento assintótico}

As propriedades estatísticas e fatos empíricos que foram discutidos no Capítulo 2 se focalizam principalmente em propriedades médias do order book (fatos estilizados), que em nosso caso agora correspondem às esperanças incondicionais que são quantidades que envolvem a medida estacionaria de $X$. A ergodicidade da cadeia de Markov $X$, mostrado na Proposição 3.4.2, implica que tais espereças $E\left[f\left(X_{\infty}\right)\right]$ podem ser calculadas simulando o modelo ao longo de um tempo muito grande $T$ e fazendo a média dos $f(X(t))$ sobre a trajetória simulada:

$$
\frac{1}{T} \int_{0}^{T} f(X(t)) d t \rightarrow E\left[f\left(X_{\infty}\right)\right] \quad \text { a.s. } \quad \text { as } \quad T \rightarrow \infty
$$

O modelo foir simulado para um grande numero de intervalos que correspondam à mesma quantidade de tempo que os dados empíricos (CST10). Nos simulamos o order book durante um longo horizonte ( $n=10^{6}$ eventos) e observamos o número médio de ordens a distâncias $1 \leq i \leq 20$ ticks do melhor cotação oposta. 


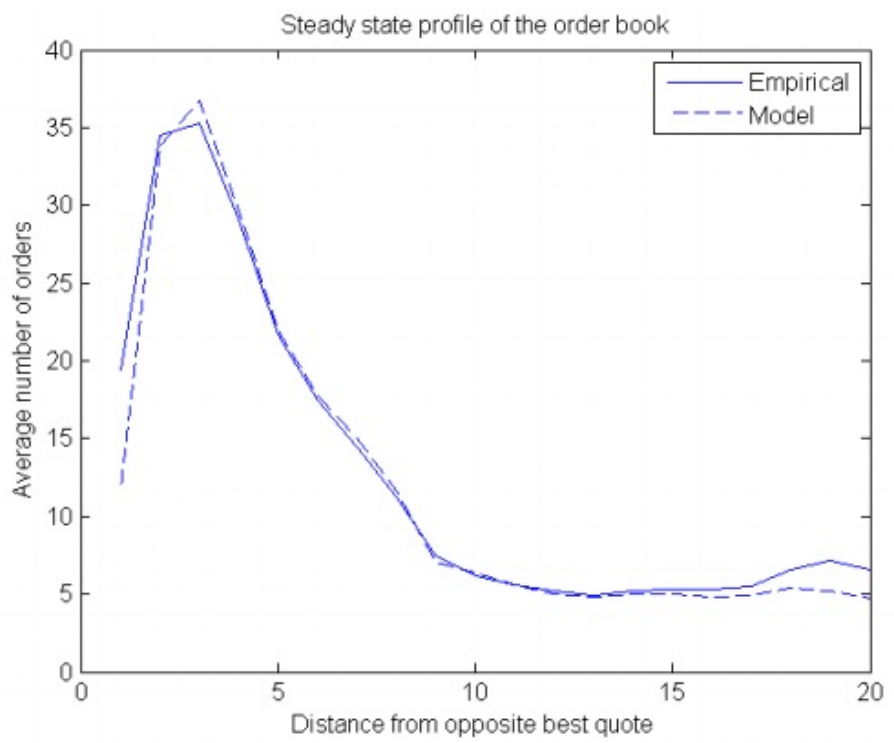

A forma do estado estacionário do order book descreve o impacto médio de mercado das negociações. A Figura 3.6 mostra o perfil médio do order book, na qual mostra uma corcova (neste caso, em dois tick do bid/ask), como foi observado em estudos empíricos (BFL08). Então o modelo assintóticamente é capaz de reproduzir muito bem o fatos estilizados.

\subsection{Conclusões do modelo}

Nos apresentamos um modelo estocástico estilizado que descreve a dinâmica do limit order book, onde as ocorrências de eventos do mercado que são os fluxos de ordens - market order, limit order e cancelamentos - são governados por processos de Poisson independentes. A formulação do modelo, que pode ser visto como um sistema de filas, é baseado totalmente em quantidades observáveis de modo que os parâmetros podem ser estimados facilmente a partir de observações de eventos no estado acutal do order book.

O modelo é simples o suficiente para permitir o calculo semi-analítico de probabilidades condicionais de vários eventos de interesse, via métodos de transformadas de Laplace, o modelo consegue reproduzir adequadamente o comportamento de curto prazo do order book. A capacidade do modelo para calcular as distribuições condicionais é útil para a previsão de corto prazo e desenho de estratégias de negociação automatizadas. Finalmente, os resultados da simulação ilustram que o modelo também apresenta factos realistas para longo prazo (estado estacionário) dado que ele pode reproduzir as propriedades estatísticas e fatos empíricos de dados order book. 


\section{Capítulo 4}

\section{Previsão de Preços para dados de nível-I na presença de liquidez oculta}

\subsection{Introdução}

O termo "Order Book" (OB) é generalmente utilizado para descrever os preços e tamanhos dos bid a ask em mercados financieros (bolsas de valores) con leiloẽs continuas, tais como NYSE-ARCA, BATS ou NASDAQ. Uma distinção importante é feita frequentemente entre os dados de nível I e de nível II, o primeiro se refere ao preço e tamanho do melhor bid/ask, e o segundo a todos os preços e tamanhos disponíveis no order book; em ambos casos, o OB fornece informação sobre a profundidade do mercado. Uma questão de interesse obvio é saber se o OB fornece informação sobre os movimentos de curto prazo dos preços, e no particular, se os dados de nível-I tem suficiente informação para fazer previções de curto prazo dos movimentos dos preços.

O modelo que será apresentado agora foi proposto por Avellaneda, Reed e Stoikov (ARS10), é um modelo simples que toma em consideração dinâmicas tipo difusão para os tamanhos do bid e ask, e centra o foco só no ask e bid como melhor preço (dados de nível I). Em contraste com o modelo apresentado anteriormente, este modela explicitamente as cotações do melhor bid e ask na presença de liquidez oculta, ou seja, os tamanhos que não são mostrados no OB mas podem influenciar nas probabilidades de um movimento ascendente do preço. A estimação da liquidez oculta permitirá medir e comparar o conteúdo de informação do order book e gerar melhores previsões.

\subsection{Objetivos do modelo}

- Fazer previsões do preços de curto prazo.

1. Dado as cotações do melhor bid/ask.

2. Dados as estatísticas sobre as taxas de chegadas das ordens.

3. Dado un único parâmetro de liquidez oculta.

- Comparar a qualidade de varios mercados em termos de conteúdo de informação.

- Estimar a liquidez oculta. 


\subsection{Modelando cotações de nível-I}

Assumimos que o spread bid-ask é um tick. Nessas condições, um dos eventos deve acontecer primeiro.

1. A fila ask é esgotada eo preço ask aumenta em um tick eo preço medio " se move para cima".

2. A fila bid é esgotada eo preço bid diminui em um tick eo preço medio "se move para baixo".

As dinâmicas que conduzem a uma mudança dos preços podem assim ser visto como uma "corrida para o fundo": a fila que chega a zero primeiro provoca a mudança do preço nessa direção.

A cadeia de Markov em tempo continuo proposta para modelar ambos filas é: $\left(X_{t}, Y_{t}\right)$ que representam o tamanho do bid e ask respectivamente.

$\mathrm{Na}$ qual as mudanças nos tamanhos ocorrem com tempos exponenciais como taxas:

$$
\begin{aligned}
& \lambda=\text { taxa de chegada das ordens no ask (bid). } \\
& \mu=\text { taxa de saida das ordens no ask (bid). } \\
& \eta=\text { taxa simultânea de chegada no bid(ask) e saida no ask(bid). } \\
& h=\text { tamanho mínimo de ordem. }
\end{aligned}
$$

Empiricamente, sabemos que os tamanhos das filas são negativamente correlacionados. Por tanto é conveniente incorporar a correlação entre as filas bid e ask neste modelo, para fazer isso foi introduzido a taxa simultânea de chegada e saida $\eta$.

Com estas convenções calculamos as médias e variâncias infinitesimales

$$
\begin{aligned}
E\left[X_{t+\Delta t}-X_{t} / X_{t}, Y_{t}\right] & =h(\lambda-\mu) \Delta t+o(\Delta t) \\
E\left[Y_{t+\Delta t}-Y_{t} / X_{t}, Y_{t}\right] & =h(\lambda-\mu) \Delta t+o(\Delta t) \\
E\left[\left(X_{t+\Delta t}-X_{t}\right)^{2} / X_{t}, Y_{t}\right] & =h^{2}(\lambda+\mu+2 \eta) \Delta t+o(\Delta t) \\
E\left[\left(Y_{t+\Delta t}-Y_{t}\right)^{2} / X_{t}, Y_{t}\right] & =h^{2}(\lambda+\mu+2 \eta) \Delta t+o(\Delta t) \\
E\left[\left(X_{t+\Delta t}-X_{t}\right)\left(Y_{t+\Delta t}-Y_{t}\right) / X_{t}, Y_{t}\right] & =h^{2}(2 \eta) \Delta t+o(\Delta t)
\end{aligned}
$$

Se $\lambda=\mu$, os desvios e as variâncias dos tamanhos das filas são dadas por

$$
\begin{aligned}
m_{x} & =m_{y}=0 \\
\sigma_{x}^{2} & =\sigma_{y}^{2}=2 h^{2}(\lambda+\eta)
\end{aligned}
$$

Então a correlação entre as filas bid e ask é dado por

$$
\rho=\frac{-\eta}{\lambda+\eta}
$$

A cantidade de nosso interesse é

$$
\operatorname{Prob} .\left\{\Delta P>o / X_{t}, Y_{t}\right\}=\operatorname{Prob} .\left\{\tau_{y}<\tau_{x} / X_{t}, Y_{t}\right\}=p\left(X_{t}, Y_{t}\right)
$$

Na qual $\tau_{x}$ e $\tau_{y}$ é a primeira vez que o tamanho bid e ask atingem zero respectivamente. Esta probabilidade será calculada usando a difusão limite. 


\subsection{A difusão limite}

Assumimos que os tamanhos médios das filas são muito maiores do que o tamanho mínimo das ordens tipicamente negociadas, ou seja $\langle X\rangle=\langle Y\rangle \gg h$.

Assumimos tambem que a frequência das ordens por unidade do tempo é alta, ou seja, $\lambda, \eta \gg 1$.

Definimos as variáveis padrão que medem os tamanhos das filas "macroscopicamente"

$$
\begin{array}{r}
x=X /\langle X\rangle, y=Y /\langle Y\rangle, \\
\sigma^{2}=\frac{2 h^{2}(\lambda+\eta)}{\langle X\rangle^{2}},
\end{array}
$$

Sobre estas premisas, pelo teorema de limite central funcional para processos de Poisson (Bil99), o proceso $\left(x_{t}, y_{t}\right)$ pode ser aproximado pela seguinte difusão

$$
\begin{aligned}
d x_{t} & =\sigma d w_{t} \\
d y_{t} & =\sigma d z_{t} \\
E(d w d z) & =\rho d t,
\end{aligned}
$$

na qual $\rho$ e $\sigma$ foram definidos em 4.3.4 e 4.4.1, e $w_{t}, z_{t}$ são movimentos brownianos padrão.

\subsection{A equação diferencial parcial}

Consideremos a função $u(x, y)=\operatorname{Prob} .\left\{\tau_{y}<\tau_{x} / x_{t}=x, y_{t}=y\right\}$ que representa a probabilidade de que o próximo movimento do preço é para cima dado os tamanhos bid e ask padronizados $(x, y)$.

Da teoria de difusão, a função $u(x, y)$ resolve a seguinte EDP

$$
\sigma^{2}\left(u_{x x}+2 \rho u_{x y}+u_{y y}\right)=0, x>0, y>0,
$$

As condições de fronteira são

$$
\begin{aligned}
& u(0, y)=0 \text { para } y>0 \\
& u(x, 0)=1 \text { para } x>0
\end{aligned}
$$

O preço muda tâo rapidamente quanto $x_{t}$ ou $y_{t}$ atingem zero.

\subsection{A liquidez oculta}

Empiricamente, a probabilidade de que o preço suba, quando o tamanho da fila ask é pequeno com relação ao tamanho da fila bid, não sempre vai tender a zero. A hipotesis é que isso acontece por duas razões: Em primer lugar, ainda tem orders nesse preço em outros mercados que evitam o aumento do preço. A segunda razão é a existência de orders ocultas ou "iceberg orders" os quais são atribuídos a algoritmos de negociação que dividem orders grandes em partes menores que reabastecem as cotações quando eles estão esgotadas. 
A continuação modelaremos tudo isso assumindo que existe uma cantidade fixa de liquidez oculta $H$ por trás das cotações bid e ask.

Isso se traduz em

$$
\sigma^{2}\left(p_{x x}+2 \rho p_{x y}+p_{y y}\right)=0, x>-H, y>-H,
$$

com condições de fronteia

$$
\begin{aligned}
& p(-H, y)=0 \text { para } y>-H \\
& p(x,-H)=1 \text { para } x>-H .
\end{aligned}
$$

Em outras palavras, podemos resolver o problema com condições de fronteira em zero e usar a relação

$$
p(x, y ; H)=u(x+H, y+H) .
$$

\subsection{Solução do EDP}

\subsubsection{Teorema}

A probabilidade de um movimento ascendente no preço médio é dado por

$$
p(x, y ; H)=u(x+H, y+H)
$$

Onde a função $u(x, y)$ satisfaze a equação $(5.1)$ e sua forma explícita é

$$
u(x, y)=\frac{1}{2}\left(\frac{1-\arctan \left(\sqrt{\frac{1+\rho}{1-\rho}} \frac{y-x}{y+x}\right)}{\arctan \left(\sqrt{\frac{1+\rho}{1-\rho}}\right)}\right)
$$

A prova deste resultado pode ser visto em (ARS10). 


\subsubsection{Observações}

- Filas não correlacionadas $(\rho=0)$, obtemos a seguente simplificação.

$$
\begin{aligned}
& \text { problema } \\
& p_{x x}+p_{y y}=0, x>-H, y>-H, \\
& \text { e } \\
& p(-H, y)=0 \text { para } y>-H, \\
& p(x,-H)=0 \text { para } x>-H, \\
& \text { solução } \\
& p(x, y ; H)=\frac{2}{\pi} \arctan \left(\frac{x+H}{y+H}\right) .
\end{aligned}
$$

- Filas perfeitamente correlacionadas negativamente $(\rho=-1)$, obtemos a seguente simplificação

$$
\begin{aligned}
& \text { problema } \\
& p_{x x}-2 p_{x y}+p_{y y}=0, x>-H, y>-H, \\
& \text { e } \\
& p(-H, y)=0 \text { para } y>-H, \\
& p(x,-H)=1 \text { para } x>-H \text {, } \\
& \text { solução } \\
& p(x, y ; H)=\frac{x+H}{x+y+2 H} .
\end{aligned}
$$

\subsection{Análise de dados}

Nesta seção estudaremos o conteúdo de informação dos dados de nível-I para os tickers QQQQ, XLF, JPM e AAPL, durante os primeiros cinco dias de negociação no 2010 (ou seja, 4-8 janeiro).

Todos os tickers são negociados em diversas bolsas (NASDAQ, NYSE e BATS), e isso nos permitirá comparar o conteúdo de informação desses mercados.

Em nosso análise dos dados, vamos-nos concentrar na liquidez oculta para o modelo de filas perfeitamente correlacionados negativamente conforme 4.7.3. Obtendremos a liquidez oculta implícita para cada ticker em cada mercado que fornecerá uma ideia de a capacidade do mercado para prever 
os movimentos dos preços de corto prazo.

A Tabela 1. contem uma pequena amostra dos dados, e na Tabela 2. apresentam-se algumas estatísticas de resumo dos ticker nos três mercados.

\begin{tabular}{llllllll}
\hline symbol & date & time & bid & ask & bsize & asize & exchange \\
QQQQ & $2010-01-04$ & $09: 30: 23$ & 46.32 & 46.33 & 260 & 242 & $\mathrm{~T}$ \\
QQQQ & $2010-01-04$ & $09: 30: 23$ & 46.32 & 46.33 & 264 & 242 & $\mathrm{~T}$ \\
QQQQ & $2010-01-04$ & $09: 30: 24$ & 46.32 & 46.33 & 210 & 271 & $\mathrm{P}$ \\
QQQQ & $2010-01-04$ & $09: 30: 24$ & 46.32 & 46.33 & 210 & 271 & $\mathrm{P}$ \\
QQQQ & $2010-01-04$ & $09: 30: 24$ & 46.32 & 46.33 & 161 & 271 & $\mathrm{P}$ \\
\hline
\end{tabular}

Tabela 4.1: Uma amostra dos dados

\begin{tabular}{lllllll}
\hline Ticker & Exchange & num quotes & quotes/sec & avg(spread) & avg(bsize+asize) & avg(price) \\
\hline \hline XLF & NASDAQ & $0.7 \mathrm{M}$ & 7 & 0.010 & 8797 & 15.02 \\
XLF & NYSE & $0.4 \mathrm{M}$ & 4 & 0.010 & 10463 & 15.01 \\
XLF & BATS & $0.4 \mathrm{M}$ & 4 & 0.011 & 7505 & 14.99 \\
\hline QQQQ & NASDAQ & $2.7 \mathrm{M}$ & 25 & 0.010 & 1455 & 46.30 \\
QQQQ & NYSE & $4.0 \mathrm{M}$ & 36 & 0.011 & 1152 & 46.27 \\
QQQQ & BATS & $1.6 \mathrm{M}$ & 15 & 0.011 & 1055 & 46.28 \\
\hline JPM & NASDAQ & $1.2 \mathrm{M}$ & 11 & 0.011 & 87 & 43.81 \\
JPM & NYSE & $0.7 \mathrm{M}$ & 6 & 0.012 & 47 & 43.77 \\
JPM & BATS & $0.6 \mathrm{M}$ & 5 & 0.014 & 39 & 43.82 \\
\hline AAPL & NASDAQ & $1.3 \mathrm{M}$ & 13 & 0.034 & 9.1 & 212.50 \\
AAPL & NYSE & $0.4 \mathrm{M}$ & 4 & 0.046 & 5.7 & 212.66 \\
AAPL & & $0.6 \mathrm{M}$ & 6 & 0.054 & 4.5 & 212.43
\end{tabular}

Tabela 4.2: Resumo estatístico

O processo para estimar a liquidez oculta $H$ é o seguente:

- Separe os dados por mercado.

- Um dia de negociação de cada vez.

- Parcelar as cotações (tamanhos do bid e ask) por deciles.

- Para cada parcela $(i, j)$, calcular as probabilidades empíricas de que o preço suba $u_{i j}$.

- Contar o número de ocorrências de cada parcela $d_{i j}$.

- Realizar um ajuste de mínimos quadrados com o modelo, i.e,

$$
\min _{H} \sum_{i, j}\left[\left(u_{i j}-\frac{i+H}{i+j+2 H}\right)^{2} d_{i j}\right]
$$

E obtemos a liquidez oculta $H$ para cada mercado e conseguiremos classificar os mercados en termos da liquidez oculta ou conte`udo de informação.

A Tabela 3. mostra a liquidez oculta estimada $H$ por mercado e por ticker.

A classificação dos mercados é a seguinte: 


\begin{tabular}{llll}
\hline Ticker & NASDAQ & NYSE & BATS \\
\hline \hline XLF & 0,15 & 0.17 & 0.17 \\
QQQQ & 0.21 & 0.04 & 0.18 \\
JPM & 0.17 & 0.17 & 0.11 \\
AAPL $\mathrm{s}=1$ & 0.16 & 0.90 & 0.65 \\
AAPL $\mathrm{s}=2$ & 0.31 & 0.60 & 0.64 \\
AAPL $\mathrm{s}=3$ & 0.31 & 0.69 & 0.63 \\
\hline
\end{tabular}

Tabela 4.3: Liquidez oculta implícita por ticker e por mercado

- Para XLF, NASDAQ tem a menor liquidez oculta.

- Para QQQQ, NYSE-ARCA tem a menor liquidez oculta.

- Para JPM, BATS tem a menor liquidez oculta.

- Para AAPL, NASDAQ tem a menor liquidez oculta. 
Os resultados do ajuste são

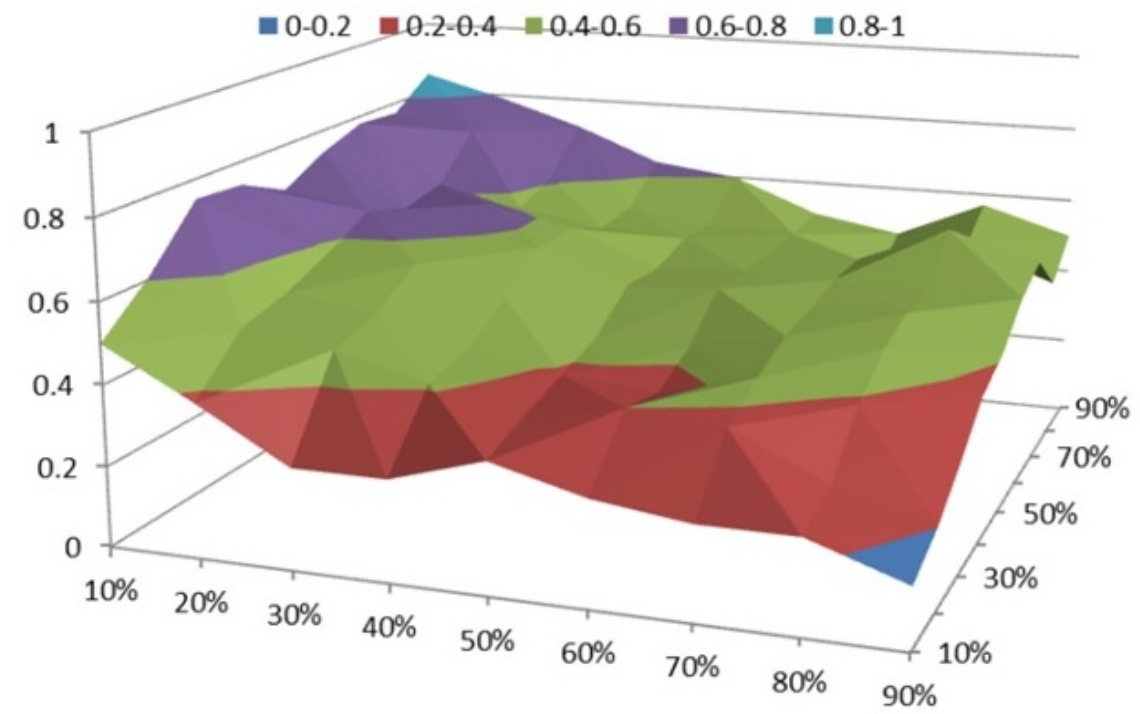

Figura 4.1: As probabilidades empíricas para movimento ascendente dos preços (XLF em NASDAQ)

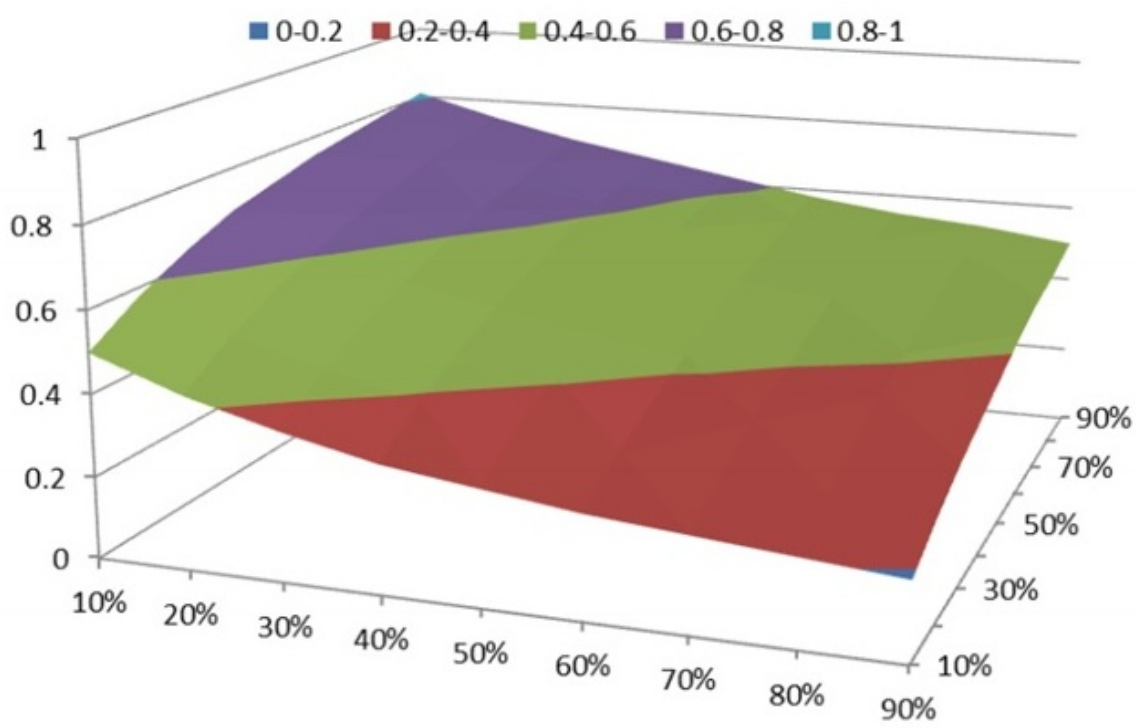

Figura 4.2: Probabilidades teóricas do modelo ajustado (XLF em NASDAQ) 


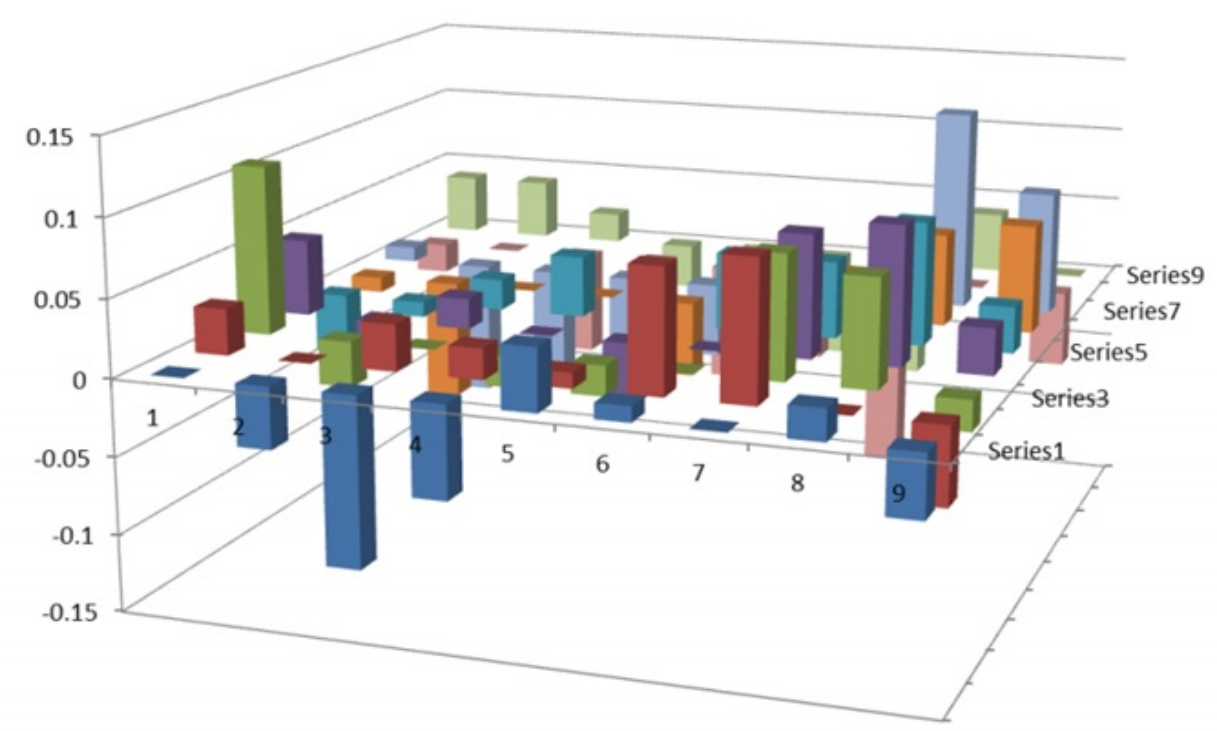

Figura 4.3: Diferença entre as probabilidades empíricas e ajustadas

\subsection{Concluções do modelo}

Com base em um modelo de difusão das orders do order book, o modelo fornece soluções de forma fechada para as probabilidades de aumentos pequenos do preço condicionado a estados nas cotações do nível-I; tais probabilidades são uma função do tamanho da fila bid, tamanho da fila ask e um parâmetro ajustável $H$ (chamado liquidez oculta).

O resultado é que o modelo permite classificar os diferentes mercados em termos de sua liquidez oculta ou, de forma equivalente, o nível de informação que tem para prever os movimentos dos preços de curto prazo; se o tamanho de liquidez oculta é pequena, dizemos que as catações são informativos em termos da capacidade de previsão. 


\section{Capítulo 5}

\section{Dinâmica do Spread bid-ask no limit order book}

\subsection{Introdução}

No order book os preços são classificados em preços de ordens de compra y preços de ordens de venda, nosso interesse neste capitulo é estudar a evolução do melhor preço de compra e melhor preço venda, preço bid/ask respectivamente, pois eles nos fornecem de informação para calcular probabilidades de eventos de grande interesse e também condicionam o comportamento assintótico do spread bid- ask o qual também esta relacionado a fatos estilizados discutidos no Capítulo 2 Nos propomos um modelo em tempo contínuo para a dinâmica dos preços bid e ask que nos permitirá estudar a evolução destes preços eo comportamento assintótico do spread. O modelo é construído de modo que reproduz os dados e comportamentos empíricos do spread no limit order book, os resultados apresentados neste capítulo são parte de uma investigação que ainda esta em adamento.

\subsection{Definição do modelo}

O preço bid/ask no tempo $t$ é denotado por $P_{-}(t) / P_{+}(t)$ e o spread bid-ask no tempo $t$ é denotado por $S(t)$, na qual $S(t)=P_{+}(t)-P_{-}(t)$.

Defina por $\xi(t)$ o par $\left(P_{-}(t), P_{+}(t)\right)$ para $t \geq 0$, onde $P_{+}(t)<P_{-}(t)$. O processo $\xi(t)$ é uma cadeia de Markov em tempo contínuo e com espaço de estados $\mathbb{Z}^{2}$. Defina também por $\eta(t)$ o par $\left(P_{-}(t), S(t)\right)$ para $t \geq 0$. O processo $\eta(t)$ é uma cadeia de Markov em tempo contínuo mas o espaço de estados é $\mathbb{Z} \times \mathbb{Z}_{+}$.

Nosso interesse é estudar o comportamento assintótico de $\xi(t)$ mas por simplicidade consideramos o processo $\eta(t)$ pelo fato de que estes dois processos contém a mesma informação.

Associado ao modelo temos a seguinte configuração dos preços disponível no limit order book $\zeta: \mathbb{Z} \rightarrow\{-1,0,1\}$. Para $t \geq 0$, e dados $P_{-}(t), P_{+}(t) \in \mathbb{Z}$ classificamos os preços em

Preços das ordens de compra

$$
\zeta(n)=-1, \quad \text { para } n \leq P_{-}(t)
$$

Preços das ordens de venda

$$
\zeta(n)=1, \quad \text { para } n \geq P_{+}(t)
$$

E os preços entre $P_{-}(t)$ e $P_{+}(t)$ (preços que ainda não tem nenhuma ordem)

$$
\zeta(n)=0, \quad \text { para } P_{-}(t)<n<P_{+}(t) .
$$


Note-se que se $S(t)=k$ para $k \geq 1$, então tem $k-1$ preços entre $P_{-}(t)$ e $P_{+}(t)$ que ainda não tem ordens. Para continuar com a analise nos propomos diferentes tipos de dinâmicas para os preços bid e ask.

\subsection{Dinâmicas dos preços bid e ask}

Nesta seção propomos diferentes tipos de dinâmicas para os preços e estudamos as propriedades assintóticas em cada caso.

Sejam $\beta_{-}, \beta_{+}$e $\alpha_{-}, \alpha_{+}$escalares positivos e finitos. Definia $\beta=\beta_{-}+\beta_{+}$e $\alpha=\alpha_{-}+\alpha_{+}$.

Assumir $S(t)=k$, para $k \in \mathbb{Z}_{+}$.

\subsubsection{Taxas de transição uniforme}

- As transições para $P_{-}(t)$ :

$$
\begin{aligned}
& P_{-}(t) \rightarrow P_{-}(t)-1, \quad \text { com taxa } \quad \beta_{-} \\
& P_{-}(t) \rightarrow P_{-}(t)+l, \quad \text { com taxa } \frac{\alpha_{-}}{k-1}
\end{aligned}
$$

onde $l \in\{1,2, \ldots, k-1\}$

- As transições para $P_{+}(t)$ :

$$
\begin{aligned}
& P_{+}(t) \rightarrow P_{+}(t)+1, \quad \text { com taxa } \quad \beta_{+} \\
& P_{+}(t) \rightarrow P_{+}(t)-l, \quad \text { com taxa } \frac{\alpha_{+}}{k-1}
\end{aligned}
$$

onde $l \in\{1,2, \ldots, k-1\}$

Nestas condições o processo $S(t)$ é uma cadeia de Markov com espaço de estados $\mathbb{Z}_{+}$e seguintes transições:

$$
\begin{array}{ccc}
S(t) \rightarrow S(t)+1, & \text { com taxa } & \beta \\
S(t) \rightarrow S(t)-l, & \text { com taxa } & \frac{\alpha}{k-1}
\end{array}
$$

onde $l \in\{1,2, \ldots, k-1\}$

Lema 5.3.1. Se os preços bid e ask têm taxas de transição uniforme 5.3.1, o spread $S(t)$ é uma cadeia de Markov ergódica.

Demonstração. Pelo Teorema 1.4 de (MP12), uma cadeia é recorrente positiva se existe uma função $f$ positiva, que tende ao infinito (chamada função de Lyapunov), uma constante $\epsilon>0$ e um subconjunto finito $F$ conteúdo no espaço de estados; de tal modo que $\Gamma f(k) \leq-\epsilon$ para todo $k \notin F$, onde $\Gamma$ é o gerador da cadeia de Markov $S(t)$.

O gerador da cadeia aplicado a uma função é dado por

$$
\Gamma f(k)=\beta[f(k-l)-f(k)]+\sum_{l=1}^{k-1}\left(\frac{\alpha}{k-1}\right)[f(k-l)-f(k)]
$$

Consideramos neste caso a $f(k)=k$, para $k \in \mathbb{Z}_{+}$como função de Lyapunov, notar que $f \uparrow \infty$. O gerador aplicado nessa função é dado por

$$
\Gamma f(k)=\beta-\frac{\alpha}{k-1} \sum_{l=1}^{k-1} l=\beta-\frac{k \alpha}{2}
$$


Dado que $\alpha$ e $\beta$ são finitos, para todo $k>\frac{2 \beta}{\alpha}$ sempre vai existir um $\epsilon>0$ tal que $\beta-\frac{k \alpha}{2}<-\epsilon$. Então como $f$ satisfaz a condição $\Gamma f(k) \leq-\epsilon$ para todo $k \notin F$, onde $F=\left\{1,2, \ldots,\left[\frac{2 \beta}{\alpha}\right]\right\}$, isso mostra que $S(t)$ é recorrente positivo. Desde que $\mathrm{S}(\mathrm{t})$ é claramente irredutível e aperiódico, resulta que $S(t)$ é ergódico.

Seja $\eta_{n}=\left(P_{n}, S_{n}\right)$ cadeia discreta correspondente aos saltos da cadeia de tempo contínuo $\eta(t)$. De acordo com o lema acima da cadeia $S_{n}$ é ergódica, seja $\pi$ a distribução estacionaria, e seja $\mu_{\pi}$ sua média.

Lema 5.3.2. Se os preços bid e ask têm taxas de transição uniforme 5.3.1.

$$
\frac{P_{-}(t)}{t} \rightarrow-\beta_{-}+\frac{\alpha_{-}}{2} \mu_{\pi} \quad \text { as } \quad t \rightarrow \infty
$$

Demonstração. Seja $N(t)$ o número de saltos da $\left(P_{n}, S_{n}\right)$.

Defina $P_{n}=\sum_{i=1}^{N(t)} \triangle\left(S_{i}\right)$ na qual

$$
\triangle_{i}=\left\{\begin{array}{cc}
0 & \text { com probabilidade } \frac{\alpha_{+}+\beta_{+}}{\alpha+\beta} \\
-1 & \text { com probabilidade } \frac{\beta_{-}}{\alpha+\beta} \\
l & \text { com probabilidade } \frac{\alpha-}{(k-1)(\alpha+\beta)}
\end{array}\right.
$$

para $l \in\{1, \ldots, k-1\}$

Para provar o lema, escrevemos

$$
\frac{P_{-}(t)}{t}=\frac{\sum_{i=1}^{N(t)} \triangle_{i}}{t}=\left(\frac{\sum_{i=1}^{N(t)} \triangle_{i}}{N(t)}\right)\left(\frac{N(t)}{t}\right)
$$

Estudamos os dois fatores separadamente, primeiro temos avaliar a convergência do primeiro fator

$$
\frac{\sum_{i=1}^{N(t)} \triangle_{i}}{N(t)}=\sum_{k=1}^{\infty}\left(\frac{\sum_{i=1}^{N(t)} \triangle_{i} \mathbb{I}_{\left\{S_{i}=k\right\}}}{N(t)}\right)=\sum_{k=1}^{\infty}\left(\frac{\sum_{i=1}^{N(t)} \triangle_{i} \mathbb{I}_{\left\{S_{i}=k\right\}}}{\sum_{i=1}^{N(t)} \mathbb{I}_{\left\{S_{i}=k\right\}}}\right)\left(\frac{\sum_{i=1}^{N(t)} \mathbb{I}_{\left\{S_{i}=k\right\}}}{N(t)}\right)
$$

Pela lei forte dos grandes números, obtemos

$$
\frac{\sum_{i=1}^{N(t)} \triangle_{i} \mathbb{I}_{\left\{S_{i}=k\right\}}}{\sum_{i=1}^{N(t)} \mathbb{I}_{\left\{S_{i}=k\right\}}} \rightarrow E\left(\triangle_{i} / S_{i}=k\right) \quad \text { as } \quad t \rightarrow \infty
$$

e

$$
\frac{\sum_{i=1}^{N(t)} \mathbb{I}_{\left\{S_{i}=k\right\}}}{N(t)} \rightarrow \pi(k) \quad \text { as } \quad t \rightarrow \infty
$$

Então com base nesses dois resultados de a convergência do primeiro fator é a seguinte

$$
\frac{\sum_{i=1}^{N(t)} \triangle_{i}}{N(t)} \rightarrow \sum_{k=1}^{\infty} E\left(\triangle_{i} / S_{i}=k\right) \pi(k)
$$

E no que se refere ao segundo factor, desde que $\{N(t), t \geq 0\}$ is a Poisson processo com taxa $\alpha+\beta$ então com probabilidade 1 [ver (Ros07)],

$$
\frac{N(t)}{t} \rightarrow \alpha+\beta \quad \text { as } \quad t \rightarrow \infty
$$


Combinando a convergência de dois fatores tem que

$$
\frac{P_{-}(t)}{t} \rightarrow(\alpha+\beta) \sum_{k=1}^{\infty} E\left(\triangle_{i} / S_{i}=k\right) \pi(k)
$$

Notamos que no caso de as taxas de transição uniforme, temo que

$$
E\left(\triangle_{i} / S_{i}=k\right)=\frac{-\beta_{-}}{\alpha+\beta}+\sum_{l=1}^{k-1} l\left(\frac{\alpha_{-}}{(k-1)(\alpha+\beta)}\right)=\frac{-\beta_{-}}{\alpha+\beta}+\frac{k \alpha_{-}}{2(\alpha+\beta)}
$$

e

$$
\sum_{k=1}^{\infty} E\left(\triangle_{i} / S_{i}=k\right) \pi(k)=\frac{-\beta_{-}}{\alpha+\beta}+\frac{\alpha_{-}}{2(\alpha+\beta)} \mu_{\pi}
$$

Então pela forte lei dos grandes números segue-se que

$$
\frac{P_{-}(t)}{t} \rightarrow-\beta_{-}+\frac{\alpha_{-}}{2} \mu_{\pi}
$$

\subsubsection{Taxas de transição com decaimento constante}

- As transições para $P_{-}(t)$ :

$$
\begin{aligned}
& P_{-}(t) \rightarrow P_{-}(t)-1, \quad \text { com taxa } \\
& P_{-}(t) \rightarrow P_{-}(t)+l, \quad \text { com taxa } \frac{\alpha_{-}}{k-l}
\end{aligned}
$$

onde $l \in\{1,2, \ldots, k-1\}$

- As transições para $P_{+}(t)$ :

$$
\begin{aligned}
& P_{+}(t) \rightarrow P_{+}(t)+1, \quad \text { com taxa } \\
& P_{+}(t) \rightarrow P_{+}(t)-l, \quad \text { com taxa } \frac{\alpha_{+}}{k-l}
\end{aligned}
$$

onde $l \in\{1,2, \ldots, k-1\}$

Nestas condições o processo $S(t)$ é uma cadeia de Markov com espaço de estados $\mathbb{Z}_{+}$e seguintes transições:

$$
\begin{aligned}
& S(t) \rightarrow S(t)+1, \quad \text { com taxa } \beta \\
& S(t) \rightarrow S(t)-l, \quad \text { com taxa } \frac{\alpha}{k-l}
\end{aligned}
$$

onde $l \in\{1,2, \ldots, k-1\}$

Lema 5.3.3. Se os preços bid e ask têm taxas de transição com decaimento constante 5.3.2, o spread $S(t)$ é uma cadeia de Markov ergódica.

Demonstração. Utilizando os mesmos argumentos que a prova anterior, nós escolhemos a $f(k)=k$, para $k \in \mathbb{Z}_{+}$como função de Lyapunov. O gerador aplicado nessa função neste caso é dado por

$$
\Gamma f(k)=\beta-\alpha \sum_{l=1}^{k-1} \frac{l}{k-l}
$$

Dado que $\sum_{l=1}^{k-1} \frac{l}{k-l}$ diverge conforme $k$ cresce, para qualquer $\alpha$ e $\beta$ finitos existe um $k$ suficientemente grande de tal forma que $\beta-\alpha \sum_{l=1}^{k-1} \frac{l}{k-l}<-\epsilon$, para algum $\epsilon>0$, a condição de recorrência positiva é satisfeita para todo $k \notin F$, onde $F=\left\{k \in \mathbb{Z}_{+}: \beta>\alpha \sum_{l=1}^{k-1} \frac{l}{k-l}\right\}$. 


\subsubsection{Taxas de transição com decaimento polinomial}

- As transições para $P_{-}(t)$ :

$$
\begin{aligned}
& P_{-}(t) \rightarrow P_{-}(t)-1, \quad \text { com taxa } \quad \beta_{-} \\
& P_{-}(t) \rightarrow P_{-}(t)+l, \quad \text { com taxa } \frac{\alpha_{-}}{l^{a}}
\end{aligned}
$$

onde $l \in\{1,2, \ldots, k-1\}$

- As transições para $P_{+}(t)$ :

$$
\begin{aligned}
& P_{+}(t) \rightarrow P_{+}(t)+1, \quad \text { com taxa } \quad \beta_{+} \\
& P_{+}(t) \rightarrow P_{+}(t)-l, \quad \text { com taxa } \frac{\alpha_{+}}{l^{a}}
\end{aligned}
$$

onde $l \in\{1,2, \ldots, k-1\}$

Nestas condições o processo $S(t)$ é uma cadeia de Markov com espaço de estados $\mathbb{Z}_{+}$e seguintes transições:

$$
\begin{aligned}
& S(t) \rightarrow S(t)+1, \quad \text { com taxa } \beta \\
& S(t) \rightarrow S(t)-l, \quad \text { com taxa } \frac{\alpha}{l^{a}}
\end{aligned}
$$

onde $l \in\{1,2, \ldots, k-1\}$

Lema 5.3.4. Se os preços bid e ask têm taxas de transição com decaimento polinomial 5.3.3, e além disso $a \leq 2$, então o espread $S(t)$ é ergódico.

Demonstração. Escolhemos a $f(k)=k$, para $k \in \mathbb{Z}_{+}$como função de Lyapunov. Neste caso o gerador aplicado nessa função es dado por

$$
\Gamma f(k)=\beta-\alpha \sum_{l=1}^{k-1} \frac{1}{l^{a-1}}
$$

Dado que $\sum_{l=1}^{\infty} \frac{1}{l^{a-1}}$ diverge para $a \leq 2$, seja $\alpha$ e $\beta$ qualquer, sempre existe um $k$ suficientemente grande de tal forma que $\beta-\alpha \sum_{l=1}^{k-1} \frac{1}{l^{a-1}}<-\epsilon$, para algum $\epsilon>0$, a condição de recorrência positiva é satisfeita para todo $k \notin F$, onde $F=\left\{k \in \mathbb{Z}_{+}: \beta>\alpha \sum_{l=1}^{k-1} \frac{1}{l^{a-1}}\right\}$.

Lema 5.3.5. Se os preços bid e ask têm taxas de transição com decaimento polinomial 5.3.3, e além disso $a>2$ e $\beta>\sum_{l=1}^{\infty} \frac{\alpha}{l^{a-1}}$, então o spread $S(t)$ é transiente.

Demonstração. Escolhemos a mesma função de Lyapunov no caso anterior $f(k)=k$, para $k \in \mathbb{Z}_{+}$. Neste caso o gerador aplicado nessa função também estará dado por

$$
\Gamma f(k)=\beta-\alpha \sum_{l=1}^{k-1} \frac{1}{l^{a-1}}
$$

Como temos a condição que $a>2$, a quantidade $\sum_{l=1}^{\infty} \frac{1}{l^{a-1}}$ converge. E também temos uma segunda condição que $\beta>\sum_{l=1}^{\infty} \frac{\alpha}{l^{a-1}}$.

Então dada a existência de um $\epsilon>0$, tal que $\beta-\alpha \sum_{l=1}^{k-1} \frac{1}{l^{a-1}}>\epsilon$, concluímos que o processo $S(t)$ é transiente. 


\section{Capítulo 6}

\section{Modelos simples de sequências de caracteres com infinito alfabeto}

\subsection{Introdução}

Consideramos a uma família de cadeias de Markov definidos nas sequências de caracteres (strings, ou palavras) com infinito alfabeto. Para alguns exemplos inspirados nos modelos de negociações em alta frequência, obtemos condições para ergodicidade, transitoriedade e recorrência nula. Para provar isso, usamos as técnicas de construção de funções Lyapunov .

\subsection{Descrição do modelo}

Considere o strings finito (sequência de símbolos ou caracteres) em tempo discreto

$$
\alpha=a_{1} a_{2} \ldots a_{n}
$$

Onde $n$ é o comprimento do string e os símbolos $a_{k} \in Z_{+}$. Com probabilidade $q(a, \emptyset)$ o último símbolo $a$ é removido, $\gamma a \rightarrow \gamma$, com probabilidade $q(a, b)$ o último símbolo $a$ muda para o símbolo $b, \gamma a \rightarrow \gamma b$, e com probabilidade $q(a, b c)$ o último símbolo $a$ é substituído por o par $b c, \gamma a \rightarrow \gamma b c$.

Seja $\tau_{a}$ o primeiro tempo aleatório na qual o comprimento do string crescrece em 1. Suponha que todas as esperanças $e_{a}=E \tau_{a}$ são finitas. As seguintes equações são válidas

$$
e_{a}=1+\sum_{b} q(a, b) e_{b}+\sum_{c, b} q(a, b c)\left(e_{b}+e_{c}\right)
$$

Definimos a matriz $M$ com elementos

$$
m_{a b}=q(a, b)+\sum_{c} q(a, b c)+\sum_{c} q(a, c b)
$$

Em forma vetorial temos

$$
\vec{e}=\overrightarrow{1}+M \vec{e}
$$

\subsection{Exemplos}

Some examples. Let denote $q_{a}=q(a, \emptyset)$

1. Caso em que $q_{a}=0$ para $a$ suficientemente grande . Por exemplo $\{1,2, \ldots$.$\} é o alfabeto, logo$ $q(a, a+1)=\lambda, q(a, a 1)=\delta$ para tudo $a, q(a, a-1)=\mu$, para $a>1$ e $q_{1}=q(1, \emptyset)=\mu$ onde $\lambda+\mu+\delta=1$. Todas as outras probabilidades de transição são 0s. Então, a matriz $M$ tem a 
forma: $m_{a, a+1}=\lambda, m_{11}=2 \delta, m_{a a}=\delta$ para $a>1, m_{21}=\delta+\mu, m_{a, a-1}=\mu$ onde $a>2$, e $m_{a 1}=\delta$ para $a>2$.

2. Caso em que $q_{a}=0, \operatorname{logo} q(a, a+1)=\lambda, q(a, a 1)=\delta$ para tudo $a$, onde $\lambda+\delta=1$. Todas as outras probabilidades de transição são 0s. Então, a matriz $M$ tem a forma: $m_{a, a+1}=\lambda$, $m_{11}=2 \delta, m_{a a}=\delta$ para $a>1$ e $m_{a 1}=\delta$ para $a>1$.

3. Caso onde $q_{a} \rightarrow 1$ quando $a \rightarrow \infty$. Se $q_{a}=1$ para un valor de $a$ suficientemente grande, Ele significa que temos de fato o alfabeto finito.

4. $q_{a}=$ const para un valor de $a$ suficientemente grande ou $q_{a} \rightarrow$ const

Consider the example 3 .

Lema 6.3.1. Considere as condiçôes do Exemplo 1

- Se $\lambda+\delta<\mu$ então a cadeia é ergódica.

- Se $\lambda+\delta>\mu$ e $\lambda<\mu-a$ cadeia é transiente; o tamanho do string cresce.

- If $\lambda>\mu$ a cadeia é transiente; o tamanho do string cresce eo valor dos últimos simbolos estão crescendo.

- $\lambda+\lambda_{1}=\mu$ - recorrência nula.

Demonstração. A função de Lyapunov neste caso será, [ver (FMM93)]

$$
f(\alpha)=a_{1}+a_{2}+\ldots+a_{n}
$$

onde $\alpha=a_{1} a_{2} \ldots a_{n}$ e $\alpha \neq \emptyset$. Então o incremento da função de Lyapunov será

$$
E\left(f\left(\xi_{t+1}\right)-f\left(\xi_{t}\right) \mid \xi_{t}=\alpha\right)=\lambda+\delta-\mu
$$

Lema 6.3.2. Considere as condiçôes do Exemplo 2, então a cadeia é transiente.

Demonstração. A função de Lyapunov será a mesma que o caso anterior, então o incremento da função de Lyapunov será,

$$
E\left(f\left(\xi_{t+1}\right)-f\left(\xi_{t}\right) \mid \xi_{t}=\alpha\right)=\lambda+\delta
$$

\subsection{Hipóteses}

No caso do alfabeto finito ( $M$ é uma matriz finita com elementos não-negativos) sempre existe um autovalor máximo $\lambda$ e autovetor $f$ com coordenadas positivas: $M f=\lambda f$. Foi provado que: [ver [FMM93)]

- ergodicidade é equivalente a $\lambda<1$

- recorrência nula a $\lambda=1$

- transiente a $\lambda>1$ 
A fim de provar isso a seguinte função Lyapounov é útil:

$$
g(\alpha)=\sum_{k=1} f_{a_{k}}
$$

onde $f$-autovetor. Seja $\xi(t)$ o estado do string no tempo $t$. Então o incremento da função Lyapunov é

$$
E(g(\xi(t+1))-g(\xi(t)) \mid \xi(t)=\gamma a)=(\lambda-1) f_{a}
$$

Raio de convergência É conhecido [ver (Sen80)], que, para uma matriz com elementes nãonegativos todas as séries

$$
\sum_{n} m_{a b}^{(n)} z^{n}
$$

convergem e divergem simultaneamente. Denote o raio comum de convergência por $R$. Para uma matriz finita $R=\lambda^{-1}$, onde $\lambda$ é o autovalor maximal. Em geral para matrizes de dimensões infinitas o autovalor maximal pode não existir.

Considere o menor principal $M_{N}$ da matriz $M$. Seja $R_{N}$ o raio de convergência de $M_{N}$. Então $R_{N+1}<R_{N}$ e $R_{N} \downarrow R$. Se a matriz $M$ é $R$-recorrente, Então existe convergência de autovalores maximales $\lambda_{N} \uparrow \lambda$.

Teorema 6.4.1. Seja $R<1$. Então para $N$ suficientemente grande $R_{N}<1$.

Existe uma classificação de matrizes de elementos não-negativos análoga à classificação de matrizes estocásticas: [ver (VJ67)]

- $R$ - transiente

- $R$ - recorrente

- $R$ - ergodico

\section{Hipóteses}

- Se $R<1$ - transiente

- Se $R<1$ ea matriz $M$ é $R$-ergodico, então a cadeia é transiente

- if $R<1$ ea matriz $M$ é $R$-transiente, então a cadeia é transiente

- if $R>1$ ea matriz $M$ é $R$-ergodico, então a cadeia é ergodica

- if $R>1$ ea matriz $M$ é $R$-transiente, então a cadeia é transiente 


\section{Apêndice A}

\section{Critérios para classificação de cadeias de Markov via funções de Lyapunov}

Considere uma cadeia de Markov homogênea $\left(\xi_{n}\right)_{n \geq 0}$ com espaço de estados $\mathcal{A}=\left\{\alpha_{i}, i \geq 0\right\}$ finito ou enumerável. As probabilidades de transição de $n$-passos podem ser denotadas por $p_{\alpha_{i} \alpha_{j}}^{(n)}$ ou, mais abreviadamente por $p_{i j}^{(n)}$. Seja $\left(\xi_{n}\right)_{n \geq 0}$ uma cadeia irredutível e aperiódica ea posição da cadeia no tempo $n$ é $\xi_{n}$.

Teorema A.0.2. A cadeia de Markov $\left(\xi_{n}\right)_{n \geq 0}$ é recorrente, se e somente se, existe uma função positiva $f(\alpha), \alpha \in \mathcal{A}$, e um conjunto finito $A$, tal que

$$
E\left[f\left(\xi_{m+1}\right)-f\left(\xi_{m}\right) / \xi_{m}=\alpha_{i}\right] \leq 0, \quad \forall \alpha_{i} \notin A
$$

e $f\left(\alpha_{j}\right) \rightarrow \infty$, quando $j \rightarrow \infty$.

Teorema A.0.3. A cadeia de Markov $\left(\xi_{n}\right)_{n \geq 0}$ é transitória, se e somente se, existe uma função positiva $f(\alpha), \alpha \in \mathcal{A}$, e um conjunto finito $A$ de tal modo que as seguintes desigualdades são satisfeitas:

$$
\begin{gathered}
E\left[f\left(\xi_{m+1}\right)-f\left(\xi_{m}\right) / \xi_{m}=\alpha_{i}\right] \leq 0, \quad \forall \alpha_{i} \notin A, \\
f\left(\alpha_{k}\right)<\inf _{\alpha_{j} \in A} f\left(\alpha_{j}\right), \text { pelo menos um } \alpha_{k} \notin A .
\end{gathered}
$$

Teorema A.0.4. (Foster) A cadeia de Markov $\left(\xi_{n}\right)_{n \geq 0}$ é ergódica, se e somente se, existe uma função positiva $f(\alpha), \alpha \in \mathcal{A}$, um número $\epsilon>0$ e um conjunto finito $A \in \mathcal{A}$, tal que

$$
\begin{gathered}
E\left[f\left(\xi_{m+1}\right)-f\left(\xi_{m}\right) / \xi_{m}=\alpha_{j}\right] \leq-\epsilon, \quad \alpha_{j} \notin A \\
E\left[f\left(\xi_{m+1}\right) / \xi_{m}=\alpha_{i}\right]<\infty, \quad \alpha_{i} \in A .
\end{gathered}
$$


APÊNDICE A 


\section{Referências Bibliográficas}

[AB98] T. Andersen e T. Bollerslev. Towards a unified framework for high and low frequency return volatility modeling. Statistica Neerlandica, 52:273-302, 1998.

[ARS10] M. Avellaneda, J. Reed e S. Stoikov. Forecasting prices in the presence of hidden liquidity. working paper, 2010.

[AW99] Abate e W. Whitt. Computing laplace transforms for numerical iversion via continued fractions. INFORMS Journal on Computing, 11(4):394-405, 1999.

[BFL08] J. P. Bouchaud, D. Farmer e F. Lillo. How market slowly digest changes in supply and demand. Elsevier: Academic Press, 2008.

[Bi199] P. Billingsley. Convergence of probability measures. Jhon Wiley and Sons, 1999.

[BMP02] J. P. Bouchaud, M. Mezard e M. Potters. Statistical properties of stock order books: empirical results and models. Quantitative finance, 2:251-256, 2002.

[Con01] R. Cont. Empirical properties of asset returns: stylized facts and statistical issues. Quantitative finance, 1:223-236, 2001.

[CST10] R. Cont, S. Stoikov e R. Talreja. A stochastic model for order book dynamics. Operations Research, 58:549-563, 2010.

$\left[\mathrm{FGL}^{+} 04\right]$ D. Farmer, L. Gillemot, F. Lillo, S. Mike e A. Sen. What really causes large price changes. Quantitative finance, 4:383-397, 2004.

[FMM93] G. Fayolle, V. A. Malyshev e M. V. Menshikov. Topics in constructive theory of countable Markov chains. Cambridge Univ. Presss, 1993.

[GCJG99] Gourieroux, Christian, J. Jasiak e Gaelle. Intra-day market activity. Journal of financial markets, 2(3):193-226, 1999.

[Has07] J. Hasbrouck. Empirical Market Microstructure. Oxford University Press, 2007.

[HBMS] Holli

eld, B., R. A. Miller e P. Sandas. Empirical analysis of limit order markets. Review of economic studies, $71(4): 10271063$, year $=2004$,

[Hua04] N. Huatsch. Modelling irregularly spaced financial data. Springer, 2004.

[MP12] M. Menshikov e D. Petritis. Explosion, implosion, and moments of passage times for continuous-time markov chains: a semimartingale approach. eprint arXiv:1202.0952, 2012 .

[Par98] C. Parlour. Price dynamics in limit order markets. Review of Financial Studies, 11:789816, 1998.

[PC98] Parlour e A. Christine. Price dynamics in limir order markets. Review of financial studies, 11(4):789-816, 1998. 
[RI09] Rosu e Ioanid. A dynamic model of the limit order book. Review of financial studies, 22:4601-4641, 2009.

[Ros07] S. M. Ross. Introduction to probability models. Elsevier: Academic Press, 2007.

[Sen80] E. Seneta. Non-negative matrices and markov chains. Springer Series in Statistics, 1980.

[SFGK03] E. Smith, D. Farmer, L. Gillemot e S. Krishnamurthy. Statistical theory of the continuous double auction. Quantitative finance, 3:481-514, 2003.

[VJ67] D. Vere-Jones. Ergodic properties of nonnegative matrices-1. Pacific Journal of mathematics, 22(2), 1967. 